This is a postprint version of the following published document:

Jiménez, S ., Fernández, F ., Borrajo, D . (2013). Integrating P lanning, Execution, a nd L earning t o I mprove $\mathrm{P}$ lan $\mathrm{E}$ xecution. Computational Intelligence, vol. $29, \mathrm{n}$ o. $1, \mathrm{p}$ p. 1-36. Avalaible in http://dx.doi.iorg/10.1111/j.1467-8640.2012.00447.x

(C) John Wiley \& Sons, Inc. 


\title{
INTEGRATING PLANNING, EXECUTION, AND LEARNING TO IMPROVE PLAN EXECUTION
}

\author{
SERGIO JimÉnEZ, FERnANDO FERnÁNDEZ, AND DANIEL BORRAJO \\ Universidad Carlos III de Madrid, \\ Madrid, Spain
}

\begin{abstract}
Algorithms for planning under uncertainty require accurate action models that explicitly capture the uncertainty of the environment. Unfortunately, obtaining these models is usually complex. In environments with uncertainty, actions may produce countless outcomes and hence, specifying them and their probability is a hard task. As a consequence, when implementing agents with planning capabilities, practitioners frequently opt for architectures that interleave classical planning and execution monitoring following a replanning when failure paradigm. Though this approach is more practical, it may produce fragile plans that need continuous replanning episodes or even worse, that result in execution dead-ends. In this paper, we propose a new architecture to relieve these shortcomings. The architecture is based on the integration of a relational learning component and the traditional planning and execution monitoring components. The new component allows the architecture to learn probabilistic rules of the success of actions from the execution of plans and to automatically upgrade the planning model with these rules. The upgraded models can be used by any classical planner that handles metric functions or, alternatively, by any probabilistic planner. This architecture proposal is designed to integrate off-the-shelf interchangeable planning and learning components so it can profi from the last advances in both field without modifying the architecture.
\end{abstract}

Keywords $\square$ cognitive architectures, relational reinforcement learning, symbolic planning.

\section{INTRODUCTION}

Symbolic planning algorithms reason about correct and complete action models to synthesize plans that attain a set of goals (Ghallab, Nau, and Traverso 20040). Specifying correct and complete action models is an arduous task. This task becomes harder in stochastic environments where actions may produce numerous outcomes with different probabilities. For example, think about simple to code actions like the unstack action from the classic planning domain Blocksworld. Unstacking the top block from a tower of blocks in a stochastic Blocksworld can make the tower collapse in a large variety of ways with different probabilities.

A different approach is to completely relieve humans of the burden of specifying planning action models. In this case, machine learning is used to automatically discover the preconditions and effects of the actions (Pasula, Zettlemoyer, and Kaelbling 2007). Action model learning requires dealing with effectively exploring the environment while learning in an incremental and online manner, similarly to Reinforcement Learning (RL) (Kaelbling, Littman, and Moore 1996). This approach is difficul to follow in symbolic planning domains because random explorations of the world do not normally discover correct and complete models for all the actions. This difficult is more evident in domains where actions may produce different effects and can lead to execution dead-ends.

As a consequence, an extended approach for implementing planning capabilities in agents consists of definin deterministic action models, obtaining plans with a classical planner, monitoring the execution of these plans and repairing them when necessary (Fox et al. 2006). Though this approach is frequently more practical, it presents two shortcomings. On the one hand, classical planners miss execution trajectories. The classical planning action model only 
considers the nominal effects of actions. So, unexpected outcomes of actions may result in undesired states or even worse in execution dead-ends. On the other hand, classical planners ignore probabilistic reasoning. Classical planners reason about the length/cost/duration of plans without considering the probability of success of the diverse trajectories that reach the goals.

In this paper, we present the Planning, Execution, and Learning Architecture (PELA) to overcome shortcomings of traditional integrations of deterministic planning and execution. PELA is based on introducing a learning component together with the planning and execution monitoring components. The learning component allows PELA to generate probabilistic rules about the execution of actions. PELA generates these rules from the execution of plans and compiles them to upgrade its deterministic planning model. The upgraded planning model extends the deterministic model with two kinds of information, state-dependent probabilities of action success and state-dependent predictions of execution dead-ends. PELA exploits the upgraded action models in future planning episodes using off-the-shelf classical or probabilistic planners.

The performance of PELA is evaluated experimentally in probabilistic planning domains. In these domains, PELA starts planning with a deterministic model—a STRIPS action model-which encodes a simplificatio of the dynamics of the environment. PELA automatically upgrades this action model as it learns knowledge about the execution of actions. The upgrade consist of enriching the initial STRIPS action model with estimates of the probability of success of actions and predictions of execution dead-ends. Finally, PELA uses the upgraded models to plan in the probabilistic domains. Results show that the upgraded models allow PELA to obtain more robust plans than a traditional integration of deterministic planning and execution.

The second section of the paper describes PELA in more detail. It shows PELA's information $\mathrm{fl} \mathrm{w}$ and the functionality of its three components: planning, execution, and learning. The third section explains how the learning component uses a standard relational learning tool to upgrade PELA's action models. The fourth section shows an empirical evaluation of PELA's performance. The fift section describes related work and, final y, the sixth section discusses some conclusions and future work.

\section{THE PELA}

PELA displays its three components in a loop: (1) Planning the actions that solve a given problem. Initially, the planning component plans with an off-the-shelf classical planner and a STRIPS-like action model $A$. This model is described in the standard planning language PDDL (Fox and Long 2003) and contains no information about the uncertainty of the world. (2) Execution of plans and classificatio of the execution outcomes. PELA executes plans in the environment and labels the actions executions according to their outcomes. (3) Learning prediction rules of the action outcomes to upgrade the action model of the planning component. PELA learns these rules from the actions performance and uses them to generate an upgraded action model $A^{\prime}$ with knowledge about the actions performance in the environment. The upgraded model $A^{\prime}$ can have two forms: $A_{c}^{\prime}$ a PDDL action model for deterministic cost-based planning over a metric that we call plan fragility or $A_{p}^{\prime}$ an action model for probabilistic planning in PPDDL (Younes et al. 2005), the probabilistic version of PDDL. In the following cycles of PELA, the planning component uses either $A_{c}^{\prime}$ or $A_{p}^{\prime}$, depending on the planner we use, to synthesize robust plans. Figure 1 shows the high-level view of this integration proposal. 


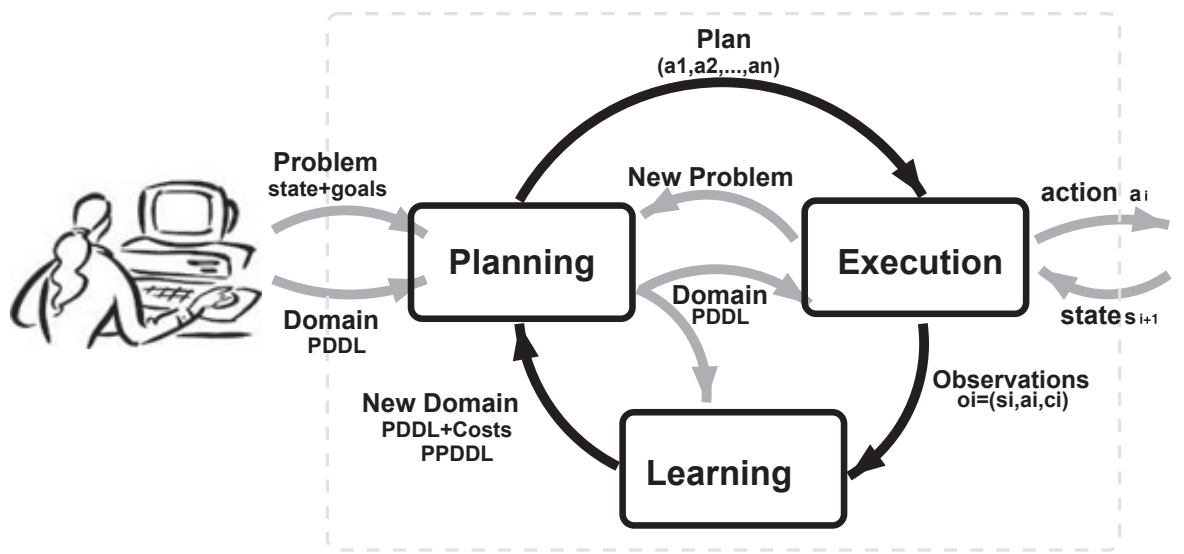

FIGURE 1. Overview of the planning, execution, and learning architecture.

The following subsections describe each component of the integration in more detail.

\subsection{Planning}

The inputs to the planning component are: a planning problem denoted by $P$ and a domain model denoted by $A$, in the firs planning episode, and by $A_{c}^{\prime}$ or $A_{p}^{\prime}$, in the subsequent ones. The planning problem $P=(I, G)$ is def ned by $I$, the set of literals describing the initial state and $G$, the set of literals describing the problem goals. Each action $a \in A$ is a STRIPSlike action consisting of a tuple ( $\operatorname{pre}(a), \operatorname{add}(a), \operatorname{del}(a))$ where $\operatorname{pre}(a)$ represents the action preconditions, $\operatorname{add}(a)$ represents the positive effects of the action and $\operatorname{del}(a)$ represents the negative effects of the action.

Each action $a \in A_{c}^{\prime}$ is a tuple (pre $(a)$,eff $(a)$ ). Again pre $(a)$ represents the action preconditions and eff $(a)$ is a set of conditional effects of the form eff $(a)=$ (and (when $c_{1}$ (and $\left.o_{1} f_{1}\right)$ ) (when $c_{2}$ (and $\left.o_{2} f_{2}\right)$ )... (when $c_{k}\left(\right.$ and $\left.o_{k} f_{k}\right)$ )) where, $o_{i}$ is the outcome of action a and $f_{i}$ is a fluen that represents the fragility of the outcome under conditions $c_{i}$. We will defin later the fragility of an action.

Each action $a \in A_{p}^{\prime}$ is a tuple ( $\operatorname{pre}(a), \operatorname{eff}(a)$ ), pre $(a)$ represents the action preconditions and $\operatorname{eff}(a)=\left(\right.$ probabilistic $\left.p_{1} o_{1} p_{2} o_{2} \ldots p_{l} o_{l}\right)$ represents the effects of the action, where $o_{i}$ is the outcome of $a$, that is, a formula over positive and negative effects that occurs with probability $p_{i}$.

The planning component synthesizes a plan $p=\left(a_{1}, a_{2}, \ldots, a_{n}\right)$ consisting of a total ordered sequence of instantiated actions. When applying $p$ to $I$, it would generate a sequence of state transitions $\left(s_{0}, s_{1}, \ldots, s_{n}\right)$ such that $s_{i}$ results from executing the action $a_{i}$ in the state $s_{i-1}$ and $s_{n}$ is a goal state, that is, $G \subseteq s_{n}$. When the planning component reasons with the action model $A$, it tries to minimize the number of actions in $p$. When reasoning with action model $A_{c}^{\prime}$, the planning component tries to minimize the value of the fragility metric. In the case of planning with $A_{p}^{\prime}$, the planning component tries to maximize the probability of reaching the goals. In addition, the planning component can synthesize a plan $p_{\text {random }}$ which contains applicable actions chosen randomly. Though $p_{\text {random }}$ does not necessarily achieve the problems goals, it allows PELA to implement different exploration/exploitation strategies. 


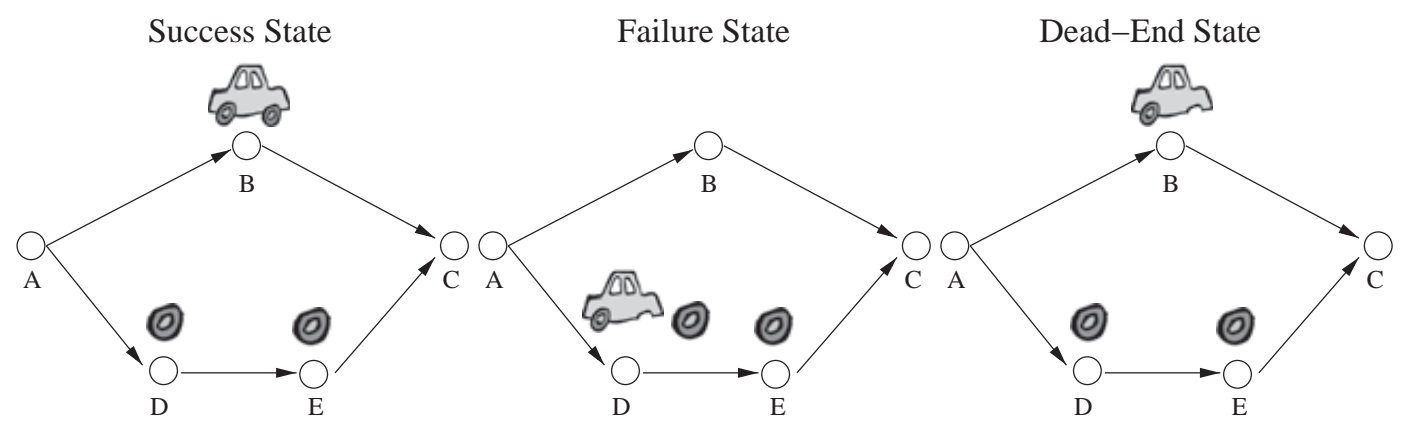

FIGURE 2. Execution episodes for the move-car action in the Tireworld.

\subsection{Execution}

The inputs to the execution component are the total ordered plan $p=\left(a_{1}, a_{2}, \ldots, a_{n}\right)$ and the initial STRIPS-like action model $A$. Both inputs are provided by the planning component. The output of the execution component is the set of observations $O=\left(o_{1}, \ldots, o_{i}, \ldots, o_{m}\right)$ collected during the executions of plans.

The execution component executes the plan $p$ one action at a time. For each executed action $a_{i} \in p$, this component stores an observation $o_{i}=\left(s_{i}, a_{i}, c_{i}\right)$, where

- $s_{i}$ is the conjunction of literals representing the facts holding before the action execution;

- $a_{i}$ is the action executed; and

- $c_{i}$ is the class of the execution. This class is inferred by the execution component from $s_{i}$ and $s_{i+1}$ (the conjunction of literals representing the facts holding after executing $a_{i}$ in $s_{i}$ ) and the STRIPS-like action model of $a_{i} \in A$. Specifical y, the class $c_{i}$ of an action $a_{i}$ executed in a state $s_{i}$ is:

-SUCCESS. When $s_{i+1}$ matches the STRIPS model of $a_{i}$ define in $A$. That is, when it is true that $s_{i+1}=\left\{s_{i} / \operatorname{Del}\left(a_{i}\right)\right\} \cup \operatorname{Add}\left(a_{i}\right)$.

-FAILURE. When $s_{i+1}$ does not match the STRIPS model of $a_{i}$ define in $A$, but the problem goals can still be reached from $s_{i+1}$; that is, the planning component can synthesize a plan that theoretically reaches the goals from $s_{i+1}$.

-DEAD - END. When $s_{i+1}$ does not match the STRIPS domain model of $a_{i}$ define in $A$, and the problem goals cannot be reached from $s_{i+1}$; that is, the planning component cannot synthesize a plan that theoretically reaches the goals from $s_{i+1}$.

Figure 2 shows three execution episodes of the action move-car (location, 10cation) from the Tireworld. In the Tireworld, a car needs to move from one location to another. The car can move between different locations via directional roads. For each movement there is a probability of getting a fla tire and fla tires can be replaced with spare ones. Unfortunately, some locations do not contain spare tires which results in execution deadends. In the example, the execution of action move-Car (A, B) could result in SUCCESS when the car does not get a fla tire or in DEAD-END when the car gets a fla tire, because at location $B$ there is no possibility of replacing fla tires. On the other hand, the execution of action move- $\operatorname{car}(A, D)$ is safer. The reason is that move-car $(A, D)$ can only result in either SUCCESS or FAILURE, because at location $D$ there is a spare tire for replacing fla tires. 


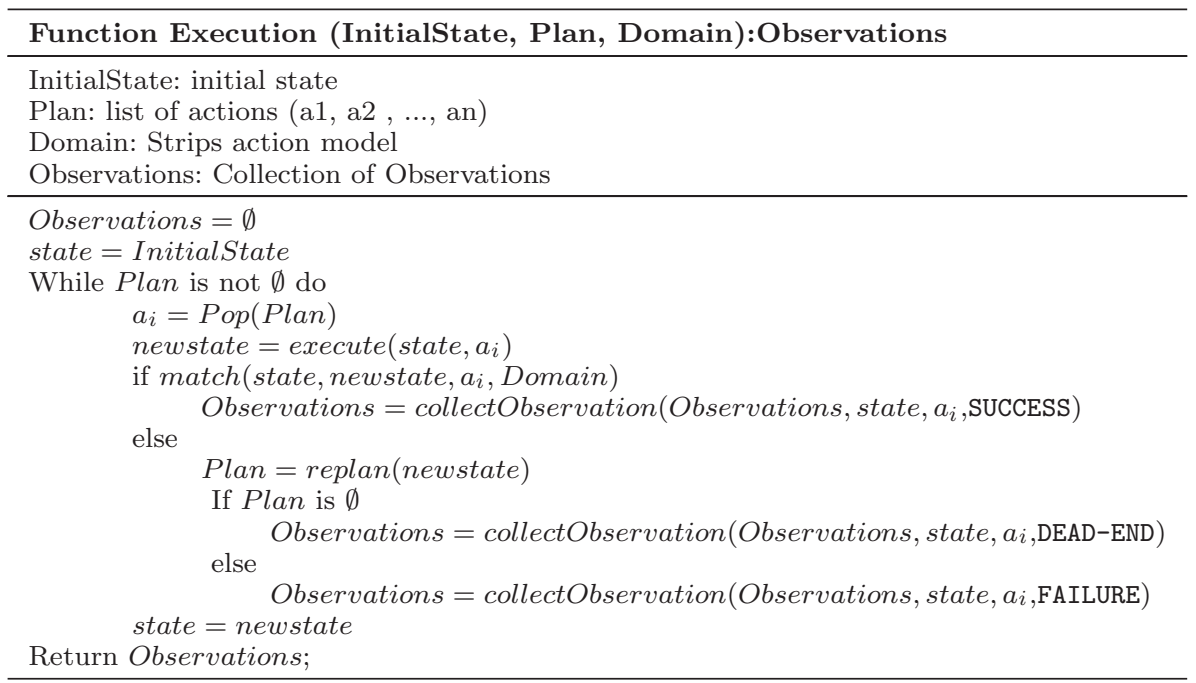

FIGURE 3. Execution algorithm for domains with dead-ends.

The algorithm for the execution component of PELA is shown in Figure 3. When the execution of an action $a_{i}$ is classifie as SUCCESS, the execution component continues executing the next action in the plan $a_{i+1}$ until there are no more actions in the plan. When the execution of an action does not match its STRIPS model, then the planning component tries to replan and provide a new plan for solving the planning problem in this new scenario. In case replanning is possible, the execution is classifie as a FAILURE and the execution component continues executing the new plan. In case replanning is impossible, the execution is classifie as a DEAD-END and the execution terminates.

\subsection{Learning}

The learning component generates rules that generalize the observed performance of actions. These rules capture the conditions referred to the state of the environment that defin each probability of execution success, failure, and dead-end of the domain actions.

Then, it compiles these rules and the STRIPS-like action model $A$ into an upgraded action model $A^{\prime}$ with knowledge about the performance of actions in the environment. The inputs to the learning component are the set of observations $O$ collected by the execution component and the original action model $A$. The output is the upgraded action model $A_{c}^{\prime}$ define in PDDL for deterministic cost-based planning or $A_{p}^{\prime}$ define in PPDDL for probabilistic planning.

Learning in PELA assumes actions have nominal effects. This is influence by the kind of actions that traditionally appear in planning tasks that typically present a unique good outcome.

The implementation of the learning component is described throughout next section.

\section{EXPLOITATION OF EXECUTION EXPERIENCE}

This section explains how PELA learns rules about the actions performance using a standard relational classifie and how PELA compiles these rules to improve the robustness 


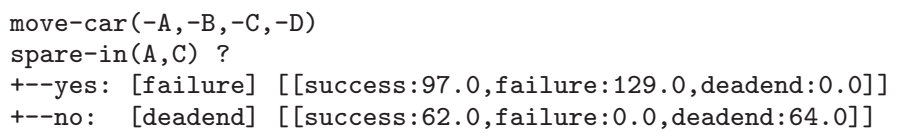

FIGURE 4. Relational decision tree for move-car (Origin, Destiny).

of the synthesized plans. In this paper, PELA focused on learning rules about the success of actions. However, the off-the-shelf spirit of the architecture allows PELA to acquire other useful execution information, like the actions durations (Lanchas et al. 2007).

\subsection{Learning Rules about the Actions Performance}

For each action $a \in A$, PELA learns a model of the performance of $a$ in terms of these three classes: SUCCESS, FAILURE, and DEAD-END. A well-known approach for multiclass classificatio consists of findin the smallest decision tree that fit a given data set. The common way to fin these decision trees is following a Top-Down Induction of Decision Trees (TDIDT) algorithm (Quinlan 1986). This approach builds the decision tree by splitting the learning examples according to the values of a selected attribute that minimize a measure of variance along the prediction variable. The leaves of the learned trees are labeled by a class value that fit the examples satisfying the conditions along the path from the root of the tree to those leaves. Relational decision trees (Blockeel and Raedt 1998) are the first-orde logic upgrade of the classical decision trees. Unlike the classical ones, relational trees work with examples described in a relational language like predicate logic. This means that each example is not described by a single feature vector but by a set of logic facts. So, the nodes of the tree do not contain tests about the examples attributes, but logic queries about the facts holding in the examples.

For each action $a \in A$, PELA learns a relational decision tree $t_{a}$. Each branch of the learned decision tree $t_{a}$ represents a prediction rule of the performance of the corresponding action $a$ :

- The internal nodes of the branch represent the set of conditions under which the rule of performance is true.

- The leaf nodes contain the corresponding class; in this case, the action performance (SUCCESS, FAILURE, or DEAD-END) and the number of examples covered by the pattern.

Figure 4 shows the decision tree learned by PELA for action move-car(Origin, Destiny) using 352 tagged examples. According to this tree, when there is a spare tire at Dest iny, the action failed 97 over 226 times, while when there is no spare tire at Dest iny, it caused an execution dead-end in 64 over 126 times.

To build a decision tree $t_{a}$ for an action $a$, the learning component receives two inputs

- The language bias specifying the restrictions in the parameters of the predicates to constrain their instantiation. This bias is automatically extracted from the STRIPS domain definition (1) the types of the target concept are extracted from the action definitio and (2) the types of the rest of literals are extracted from the predicates definition Predicates are extended with an extra parameter called example that indicates the identifie of the observation. Besides, the parameters list of actions is also augmented with a label that 


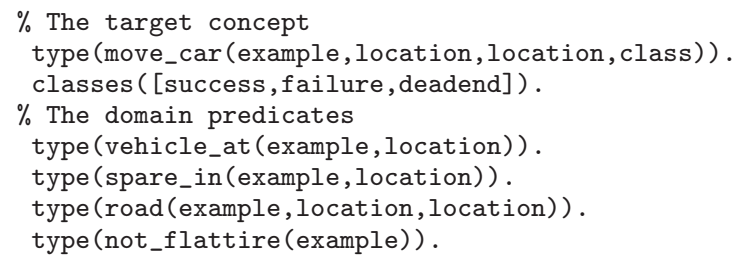

FIGURE 5. Language bias for the Tireworld.

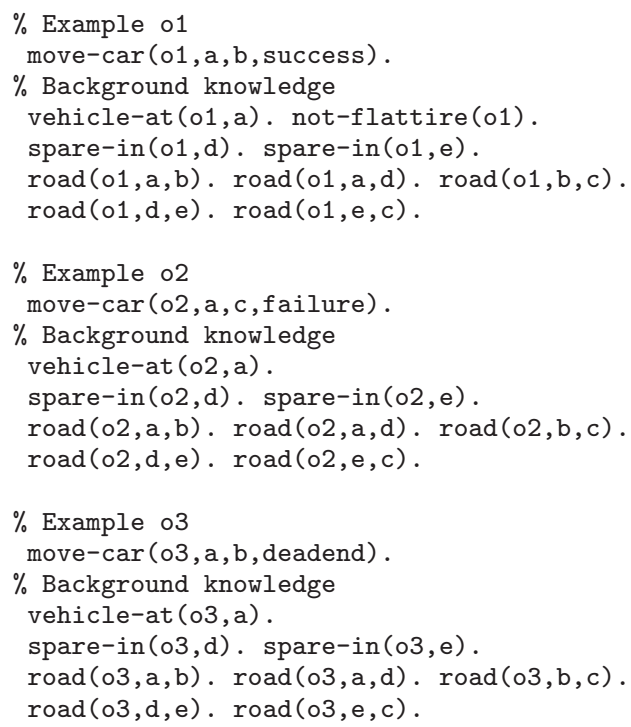

FIGURE 6. Knowledge base after the executions of Figure 2.

describes the class of the learning example (SUCCESS, FAILURE, or DEAD-END). Figure 5 shows the language bias specifie for learning the model of performance of action move-car (Origin, Destiny) from the Tireworld.

- The knowledge base, specifying the set of examples of the target concept, and the background knowledge. In PELA, both are automatically extracted from the observations collected by the execution component. The action execution (example of target concept) is linked with the state literals (background knowledge) through the identifie of the execution observation. Figure 6 shows a piece of the knowledge base for learning the patterns of performance of the action move-car (Origin, Destiny). Particularly, this example captures the execution examples with identifie $\circ 1,02$, and $\circ 3$ that resulted in success, failure and dead-end respectively, corresponding to the action executions of Figure 2.

PELA uses TILDE ${ }^{1}$ for the tree learning but there is nothing that prevents from using any other relational decision tree learning tool.

${ }^{1}$ TILDE (Blockeel and Raedt 1998) is a relational implementation of the Top-Down Induction of Decision Trees (TDIDT) algorithm (Quinlan 1986). 


\subsection{Upgrade of the Action Model with Learned Rules}

PELA compiles the STRIPS-like action model $A$ and the learned trees into an upgraded action model. PELA implements two different upgrades of the action model: (1) compilation to a metric representation; and (2) compilation to a probabilistic representation. Next, there is a detailed description of the two compilations.

3.2.1. Compilation to a Metric Representation. In this compilation, PELA transforms each action $a \in A$ and its corresponding learned tree $t_{a}$ into a new action $a^{\prime} \in A_{c}^{\prime}$ which contains a metric of the fragility of $a$. The aim of the fragility metric is making PELA generate more robust plans that solve more problems in stochastic domains. This aim includes two tasks, avoiding execution dead-ends and avoiding replanning episodes, that is, maximizing the probability of success of plans. Accordingly, the fragility metric expresses two types of information: it assigns infinit cost to situations that can cause execution dead-ends and it assigns a cost indicating the success probability of actions when they are not predicted to cause execution dead-ends.

Given $\operatorname{prob}\left(a_{i}\right)$ as the probability of success of action $a_{i}$, the probability of success of a total ordered plan $p=\left(a_{1}, a_{2}, \ldots, a_{n}\right)$ can be define as

$$
\operatorname{prob}(p)=\prod_{i=1}^{n} \operatorname{prob}\left(a_{i}\right) .
$$

Intuitively, taking the maximization of $\operatorname{prob}(p)$ as a planning metric should guide planners to fin robust solutions. However, planners do not efficient y deal with a product maximization. So, despite this metric is theoretically correct, experimentally it leads to poor results in terms of solutions quality and computational time. Instead, existing planners are better designed to minimize a sum of values (like length/cost/duration of plans). This compilation define a metric indicating not a product maximization but a sum minimization, so off-the-shelf planners can use it to fin robust plans. The definitio of this metric is based on the following property of logarithms

$$
\log \left(\prod_{i} x_{i}\right)=\sum_{i} \log \left(x_{i}\right) .
$$

Specificaly, we transform the probability of success of a given action into an action cost called fragility. The fragility associated to a given action $a_{i}$ is computed as

$$
\operatorname{fragility}\left(a_{i}\right)=-\log \left(\operatorname{prob}\left(a_{i}\right)\right) \text {. }
$$

The fragility associated to a total ordered plan is computed as

$$
\operatorname{fragility}(p)=\sum_{i=1}^{n} \operatorname{fragility}\left(a_{i}\right) .
$$

Note that a minus sign is introduced in the fragility definitio to transform the maximization into a minimization. In this way, the maximization of the product of success probabilities along a plan is transformed into a minimization of the sum of the fragility costs.

Formally, the compilation is carried out as follows. Each action $a \in A$ and its corresponding learned tree $t_{a}$ are compiled into a new action $a^{\prime} \in A_{c}^{\prime}$ where

(i) The parameters of $a^{\prime}$ are the parameters of $a$.

(ii) The preconditions of $a^{\prime}$ are the preconditions of $a$. 


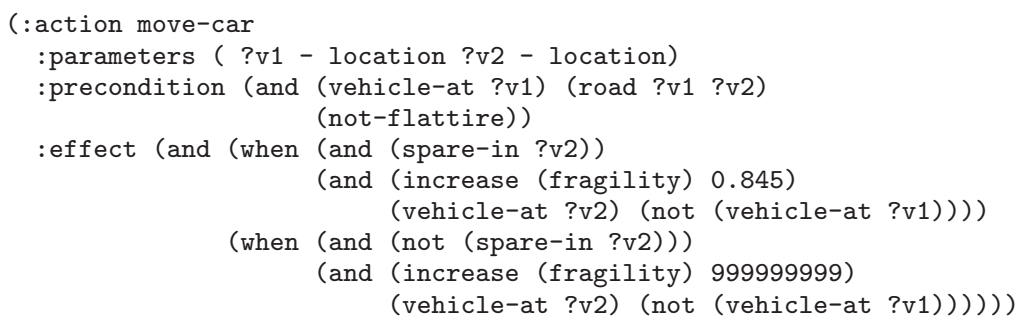

FIGURE 7. Compilation into a metric representation.

(iii) The effects of $a^{\prime}$ are computed as follows. Each branch $b_{j}$ of the tree $t_{a}$ is compiled into a conditional effect $c e_{j}$ of the form $c e_{j}=\left(\right.$ when $\left.B_{j} E_{j}\right)$ where

(i) $B_{j}=$ (and $b_{j 1} \ldots b_{j m}$ ), where $b_{j k}$ are the relational tests of the internal nodes of branch $b_{j}$ (in the tree of Figure 4 there is only one test, referring to sparein $(A, C))$;

(ii) $E_{j}=\left(\right.$ and $\left\{\operatorname{effects}(a) \cup\left(\right.\right.$ increase $\left(\right.$ fragility) $\left.\left.\left.f_{j}\right)\right\}\right)$;

(iii) $\operatorname{effects}(a)$ are the STRIPS effects of action $a$; and

(iv) (increase (fragility) $f_{j}$ ) is a new literal which increases the fragility metric in $f_{j}$ units. The value of $f_{j}$ is computed as

- when $b_{j}$ does not cover execution examples resulting in dead-ends,

$$
f_{j}=-\log \left(\frac{1+s}{2+n}\right),
$$

where $s$ refers to the number of execution examples covered by $b_{j}$ resulting in success, and $n$ refers to the total number of examples that $b_{j}$ covers. Regarding the Laplace's rule of succession we add 1 to the success examples and 2 to the total number of examples. Therefore, we assign a probability of success of 0.5 to actions without observed executions;

- when $b_{j}$ covers execution examples resulting in dead-ends.

$$
f_{j}=\infty \text {. }
$$

PELA considers as execution dead-ends states where goals are unreachable from them. PELA focuses on capturing undesired features of the states that cause dead-ends to include them in the action model. For example, in the triangle-tireworld moving to locations that do not contain spare-wheels. PELA assigns an infinit fragility to the selection of actions in these undesired situations so the generated plans avoid them because of their high cost. PELA does not capture undesired features of goals because the PDDL and PPDDL languages do not allow to include goals information in the action models.

Figure 7 shows the result of compiling the decision tree of Figure 4 . In this case, the tree is compiled into two conditional effects. Given that there is only one test on each branch, each new conditional effect will only have one condition (spare-in or not(spare-in)). As it does not cover dead-end examples, the firs branch increases the fragility cost in $-\log \left(\frac{97+1}{97+129+2}\right)$. The second branch covers dead-end examples, so it increases the fragility cost in $\infty$ (or a sufficient y big number in practice; 999,999,999 in the example). 


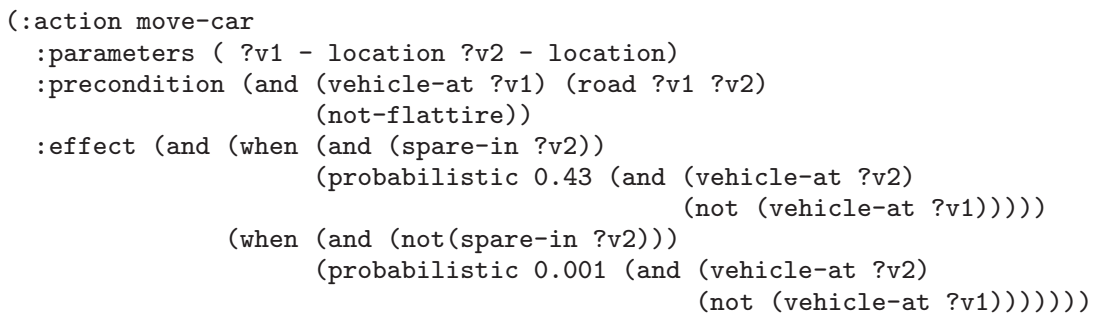

FIGURE 8. Compilation into a probabilistic representation.

3.2.2. Compilation to a Probabilistic Representation. In this case, PELA compiles each action $a \in A$ and its corresponding learned tree $t_{a}$ into a new probabilistic action $a^{\prime} \in A_{p}^{\prime}$ where

(i) The parameters of $a^{\prime}$ are the parameters of $a$.

(ii) The preconditions of $a^{\prime}$ are the preconditions of $a$.

(iii) Each branch $b_{j}$ of the learned tree $t_{a}$ is compiled into a probabilistic effect $p e_{j}=$ (when $B_{j} E_{j}$ ) where

(i) $\quad B_{j}=\left(\right.$ and $\left.b_{j 1} \ldots b_{j m}\right)$, where $b_{j k}$ are the relational tests of the internal nodes of branch $b_{j}$;

(ii) $E_{j}=\left(\right.$ probabilistic $p_{j}$ effects $\left.(a)\right)$;

(iii) effects $(a)$ are the STRIPS effects of action $a$; and

(iv) $p_{j}$ is the probability value and it is computed as

- when $b_{j}$ does not cover execution examples resulting in dead-ends,

$$
p_{j}=\frac{1+s}{2+n},
$$

where $s$ refers to the number of success examples covered by $b_{j}$, and $n$ refers to the total number of examples that $b_{j}$ covers. The probability of success is also computed following the Laplace's rule of succession to assign a probability of 0.5 to actions without observed executions;

- when $b_{j}$ covers execution examples resulting in dead-ends,

$$
p_{j}=0.001 \text {. }
$$

Again, PELA does not only try to optimize the probability of success of actions but it also tries to avoid execution dead-ends. Probabilistic planners will try to avoid selecting actions in states that can cause execution dead-ends because of their low success probability.

Figure 8 shows the result of compiling the decision tree of Figure 4 corresponding to the action move-car (Origin, Destiny). In this compilation, the two branches are coded as two probabilistic effects. The firs one does not cover dead-end examples so it has a probability of $\frac{97+1}{97+129+2}$. The second branch covers dead-end examples so it has a probability of 0.001 . 


\section{EVALUATION}

To evaluate PELA, we use the methodology define at the probabilistic track of the International Planning Competition (IPC). This methodology consists of

- A common representation language. PPDDL was define as the standard input language for probabilistic planners.

- A simulator of stochastic environments. $M D P \operatorname{sim}^{2}$ was developed to simulate the execution of actions in stochastic environments. Planners communicate with MDPsim in a high level communication protocol that follows the client-server paradigm. This protocol is based on the exchange of messages through TCP sockets. Given a planning problem, the planner sends actions to MDPsim, MDPsim executes these actions according to a given probabilistic action model described in PPDDL and sends back the resulting states.

- A performance measure. At IPC probabilistic planners are evaluated regarding these metrics:

(i) Number of problems solved. The more problems a planner solves, the better the planner performs. This is the main criterion to evaluate the performance of PELA in our experiments. In stochastic domains, planners need to avoid executions dead-ends and to reduce the number of replanning episodes to succeed reaching the problem goals in the given time bound.

(ii) Time invested to solve a problem. The less time a planner needs, the better the planner performs. Our experiments also report this measure to distinguish the performance of planners when planners solve the same number of problems.

(iii) Number of actions to solve a problem. The less actions a planner needs, the better the planner performs. Though this metric is computed at IPC, we do not use it to evaluate the performance of PELA Comparing probabilistic planners with this metric might be confusing. In some cases robust plans are the shortest ones. In other cases, longer plans are the most robust because they avoid execution dead-ends or because they have a higher probability of success. Like the time-invested metric, the number of actions could also be used to distinguish the performance of planners when they solve the same number of problems.

In our experiments both PELA and MDPSim share the same problem descriptions. However, they have different action models. On the one hand, PELA tries to solve the problems starting with a STRIPS-like description of the environment $A$ which ignores the probability of success of actions. On the other hand, MDPSim simulates the execution of actions according to a PPDDL model of the environment $A_{\text {perfect }}$. As execution experience is available PELA will learn new action models $A_{c}^{\prime}$ or $A_{p}^{\prime}$ that approach the performance of PELA to the performance of planning with the perfect model of the environment $A_{\text {perfect }}$.

\subsection{The Domains}

We evaluate PELA over a set of probabilistically interesting domains. A given planning domain is considered probabilistically interesting (Little and Thiébaux 2007) when the shortest solutions to the domain problems do not correspond to the solutions with the highest probability of success. Given that classical planners prefer short plans, a classical

${ }^{2}$ MDPsim can be freely downloaded at http://icaps-conference.org/ 
replanning approach fails more often than a probabilistic planner. These failures mean extra replanning episodes which usually involve more computation time. And/or when the shortest solutions to the domain problems present execution dead-ends. Given that classical planners prefer short plans, a classical replanning approach solves less problems than a probabilistic planner.

Probabilistically interesting domains can be generated from classical domains by increasing their robustness diversity, that is, the number of solution plans with different probability of success. In this paper, we propose to artificial y increase the robustness diversity of a classical planning domain following any of the proposed methods

- Cloning actions. Cloned actions of diverse robustness are added to the domain model. Particularly, a cloned action $a^{\prime}$ keeps the same parameters and preconditions of the original action $a$ but presents (1) different probability of success and/or (2) a certain probability of producing execution dead-ends. Given that classical planners handle STRIPS-like action models, they do not reason about the probability of success of actions and they arbitrarily choose among cloned actions ignoring their robustness.

- Adding fragile macro-actions. A macro-action $a^{\prime}$ with (1) low probability of success and/or (2) with a certain probability of producing execution dead-ends is added to the domain. Given that classical planners ignore robustness and prefer short plans, they tend to select the fragile macro-actions though they are less likely to succeed.

- Transforming action preconditions into success preferences. Given an action with the set of preconditions $p$ and effects $e$, a precondition $p_{i} \in p$ is removed and transformed into a condition for $e$ that (1) increases the probability of success and/or (2) avoids execution dead-ends. For example, when $p_{i}$ (probability $0.9\left(\right.$ and $\left.e_{1}, \ldots, e_{i}, \ldots, e_{n}\right)$ ) and when $\neg p_{i}\left(\right.$ probability 0.1 (and $\left.e_{1}, \ldots, e_{i}, \ldots, e_{n}\right)$ ). Again, classical planners prefer short plans, so they skip the satisfaction of these actions conditions though they produce plans more likely to fail.

We test the performance of PELA over the following set of probabilistically interesting domains

Blocksworld. This domain is the version of the classical four-actions Blocksworld introduced at the probabilistic track of IPC-2006. This version extends the original domain with three new actions that manipulate towers of blocks at once. Generally, off-the-shelf classical planners prefer manipulating towers because it involves shorter plans. However, these new actions present high probability of failing and causing no effects.

Slippery-gripper (Pasula et al. 2007). This domain is a version of the four-actions Blocksworld which includes a nozzle to paint the blocks. Painting a block may wet the gripper, which makes it more likely to fail when manipulating blocks. The gripper can be dried to move blocks safer. However, off-the-shelf classical planners will generally skip the dry action, because it involves longer plans.

Rovers. This domain is a probabilistic version of the IPC-2002 Rovers domain specifically define for the evaluation of PELA. The original IPC-2002 domain was inspired by the planetary Rovers problem. This domain requires that a collection of rovers equipped with different, but possibly overlapping, sets of equipment, navigate a planet surface, fin samples and communicate them back to a lander. In this new version, the navigation of rovers between two waypoints can fail. Navigation fails more often when waypoints are not visible and even more when waypoints are not marked as traversable. Off-the-shelf classical planners ignore that navigation may fail at certain waypoints, so their plans fail more often.

OpenStacks. This domain is a probabilistic version of the IPC-2006 OpenStacks domain. The original IPC-2006 domain is based on the minimum maximum simultaneous open 
TABLE 1. Topology of the Domains Chosen for the Evaluation of PELA.

\begin{tabular}{lcc}
\hline & Probabilistic & State-dependent + Probabilistic \\
\hline Dead-ends free & Blocksworld & Slippery-gripper, Rovers \\
Dead-ends presence & OpenStacks & Triangle-tireworld, Satellite \\
\hline
\end{tabular}

stacks combinatorial optimization problem. In this problem, a manufacturer has a number of orders. Each order requires a given combination of different products and the manufacturer can only make one product at a time. Additionally, the total quantity required for each product is made at the same time (changing from making one product to making another requires a production stop). From the time that the firs product included in an order is made to the time that all products included in the order have been made, the order is said to be open and during this time it requires a stack (a temporary storage space). The problem is to plan the production of a set of orders so that the maximum number of stacks simultaneously used, or equivalently, the number of orders that are in simultaneous production, is minimized. This new version, specifical y define for the evaluation of PELA, extends the original one with three cloned setup-machine actions and with one macro-action setup-machine-make-product that may produce execution dead-ends. Off-the-shelf classical planners ignore the robustness of the cloned setup-machine actions. Besides, they tend to use the setup-machine-make-product macro-action because it produces shorter plans.

Triangle-tireworld (Little and Thiébaux 2007). In this version of the tireworld both the origin and the destination locations are at the vertex of an equilateral triangle, the shortest path is never the most probable one to reach the destination, and there is always a trajectory where execution dead-ends can be avoided. Therefore, an off-the-shelf planner using a STRIPS action model will generally not take the most robust path.

Satellite. This domain is a probabilistic version of the IPC-2002 domain define for the evaluation of PELA. The original domain comes from the satellite observation scheduling problem. This domain involves planning a collection of observation tasks between multiple satellites, each equipped with slightly different capabilities. In this new version, a satellite can take images without being calibrated. Besides, a satellite can be calibrated at any direction. The plans generated by off-the-shelf classical planners in this domain skip calibration actions because they produce longer plans. However, calibrations succeed more often at calibration targets and taking images without a calibration may cause execution dead-ends.

With the aim of making the analysis of results easier, we group the domains according to two dimensions, the determinism of the action success and the presence of execution dead-ends. Table 1 shows the topology of the domains chosen for the evaluation of PELA.

- Action success. This dimension values the complexity of the learning step. When probabilities are not state-dependent one can estimate their value counting the number of success and failure examples. In this regard, it is more complex to correctly capture the success of actions in domains where action success is state-dependent.

- Execution dead-ends. This dimension values the difficult of solving a problem in the domain. When there are no execution dead-ends the number of problems solved is only affected by the combinatorial complexity of the problems. However, when there are execution dead-ends the number of problems solved depends also on the avoidance of these dead-ends. 


\subsection{Correctness of the PELA Models}

This experiment evaluates the correctness of the action models learned by PELA. The experiment shows how the error of the learned models varies with the number of learning examples. Note that this experiment focuses on the exploration of the environment and does not report any exploitation of the learned action models for problem solving. The offlin integration of learning and planning is described and evaluated later in the paper, at Section 4.3. Moreover this experiment does not use the learned models for collecting new examples. The online integration of exploration and exploitation in PELA is described and evaluated at Section 4.4 .

The experiment is designed as follows: For each domain, PELA addresses a set of randomly generated problems and learns a new action model after every twenty actions executions. Once a new model is learned it is evaluated computing the absolute error between (1) the probability of success of actions in the learned model and (2) the probability of success of actions in the true model, which is the PPDDL model of the MDPsim simulator. The probability of success of an action indicates the probability of producing the nominal effects of the action. Recall that our approach assumes actions have nominal effects. Because the probability of success may be state-dependent, each error measure is computed as the mean error over a test set of 1,000 states. $^{3}$ In addition, the experiment reports the absolute deviation of the error measures from the mean error. These deviations - shown as square brackets - are computed after every 100 actions executions and represent a confidenc estimate for the obtained measures.

The experiment compares four different exploration strategies to automatically collect the execution experience

(i) FF: Under this strategy, PELA collects examples executing the actions proposed by the classical planner Metric-FF (Hoffmann 2003).

This planner implements a deterministic forward-chaining search. The search is guided by a domain independent heuristic function which is derived from the solution of a relaxation of the planning problem.

In this strategy, when the execution of a plan yields an unexpected state, FF replans to fin a new plan for this state.

(ii) $L P G$ : In this strategy, examples are collected executing the actions proposed by the classical planner LPG (Gerevini, Saetti, and Serina 2003).

LPG implements a stochastic search scheme inspired by the SAT solver Walksat. The search space of LPG consists of "action graphs" representing partial plans. The search steps are stochastic graph modification transforming an action graph into another one.

This stochastic nature of LPG is interesting for covering a wider range of the problem space. Like the previous strategy, LPG replans to overcome unexpected states.

(iii) $L P G-\varepsilon$ Greedy: With probability $\varepsilon$, examples are collected executing the actions proposed by LPG. With probability $(1-\varepsilon)$, examples are collected executing an applicable action chosen randomly. For this experiment the value of $\varepsilon$ is 0.75 .

(iv) Random: In this strategy examples are collected executing applicable actions chosen randomly.

\footnotetext{
${ }^{3}$ The 1,000 test states are extracted from randomly generated problems. Half of the test states are generated with random walks and the other half with walks guided by LPG plans, because as shown experimentally, in some planning domains random walks provide poor states diversity given that some actions end up unexplored.
} 


random
Ipgegreedy $\ldots \ldots \ldots \ldots \ldots$

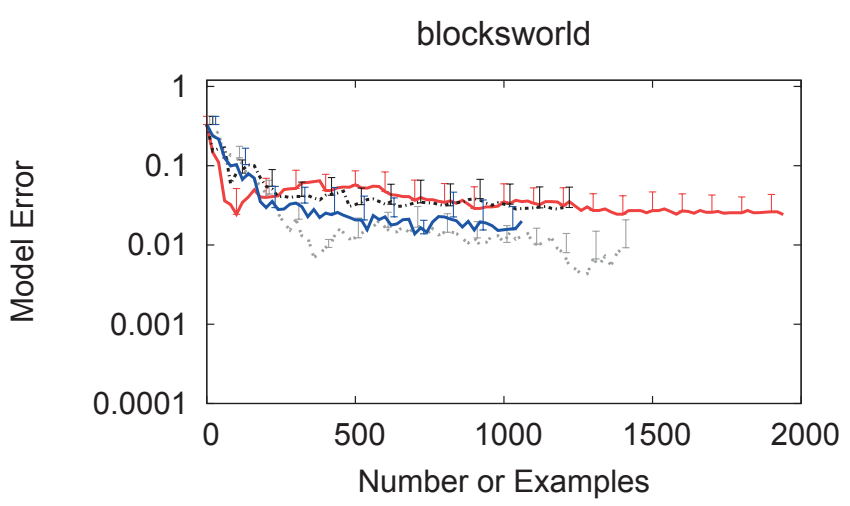

FIGURE 9. Error of the learned models in the Blocksworld domain.

In the Blocksworld domain, all actions are applicable in most of the state configurations As a consequence, the four strategies explore well the performance of actions and achieve action models with low error rates and low deviations. Despite the set of training problems is the same for the four strategies, the random strategy generates more learning examples because it is not able to solve problems. Consequently, the random strategy exhausts the limit of actions per problem. The training set for this domain consisted of 45-blocks problems. Figure 9 shows the error rates and their associated deviations obtained when learning models for the actions of the Blocksworld domain. Note that the plotted error measures may not be within the deviation intervals because the intervals are slightly shifted in the X-axis for improving their readability.

In the Slippery-gripper, there are differences in the speed of convergence of the different strategies. Specifical y, pure planning strategies FF and LPG converge slower. In this domain, the success of actions depends on the state of the gripper (wet or dry). Capturing this knowledge requires examples of action executions under both types of contexts, that is, actions executions with a wet gripper and with a dry gripper. However, pure planning strategies FF and LPG present poor diversity of contexts because they skip the action dry as it means longer plans.

In the Rovers domain the random strategy does not achieve good error rates because this strategy does not explore the actions for data communication. The explanation of this effect is that these actions only satisfy their preconditions with a previous execution of actions navigate and take-sample. Unfortunately, randomly selecting this sequence of actions with the right parameters is very unlikely. Figure 10 shows error rates obtained when learning the models for the Slippery-gripper and the Rovers domain. The training set for the Slippery-gripper consisted of 45-blocks problems. The training set for the Rovers domain consisted of 60 problems of 10 locations and 3 objectives.

In the Openstacks domain pure planning strategies (FF and LPG) prefer the macro-action for making products despite it produces dead-ends. As a consequence, the original action for making products ends up being unexplored. As shown by the $L P G-\varepsilon G r e e d y$ strategy, this negative effect is relieved including extra stochastic behavior in the planner. On the other hand, a full random strategy ends up with some actions unexplored as happened in the Rovers domain. 

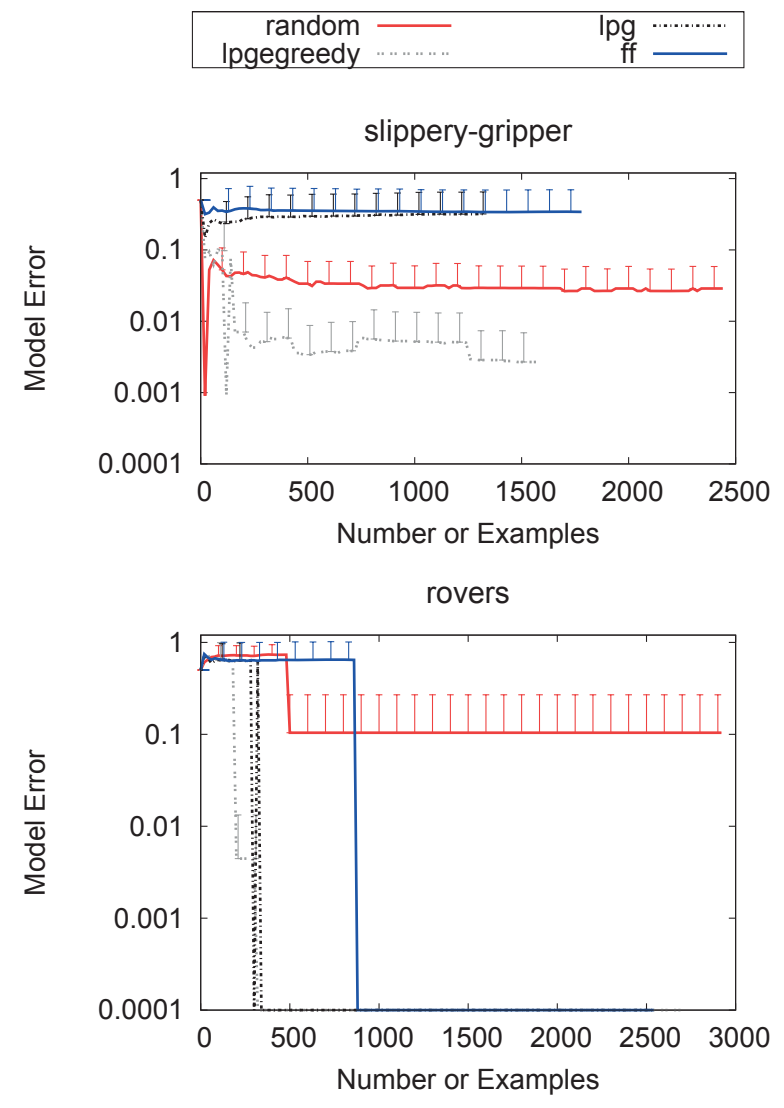

FIGURE 10. Model error in the Slippery-gripper and Rovers domains.

In the Triangle-tireworld domain, error rates fluctuat roughly because the action model consists only of two actions. In this domain the FF strategy does not reach good error rates because the shortest path to the goals always lack of spare-tires. The performance of the FF strategy could be improved by initially placing the car in diverse locations of the triangle. Figure 11 shows error rates obtained for the Openstacks and the Triangle-tireworld domain. The training set for the Openstacks consisted of 100 problems of 4 orders, 4 products, and 6 stacks. The training set for the Triangle-tireworld consisted of 100 problems of size 5 .

For the Satellite domain, we used two sets of training problems. The firs one was generated with the standard problem generator provided by IPC. Accordingly, the goals of these problems always are either have-image or pointing. Given that in this version of the satellite domain can have images without calibrating, the action calibrate was only explored by the random strategy. However, the random strategy cannot explore action take-image because it implies a previous execution of actions switch-on and turnto with the right parameters. To avoid these effects and guaranteeing the exploration of all actions, we built a new problem generator that includes as goals any dynamic predicate of the domain. Figure 12 shows the results obtained when learning the models with the two different training sets. As shown in the graph titled satellite2, the second set of training problems improves the exploration of the configuration guided by planners and achieves models of a higher quality. The training set for the satellite domain consisted of 60 problems with 1 satellite and 4 objectives. 

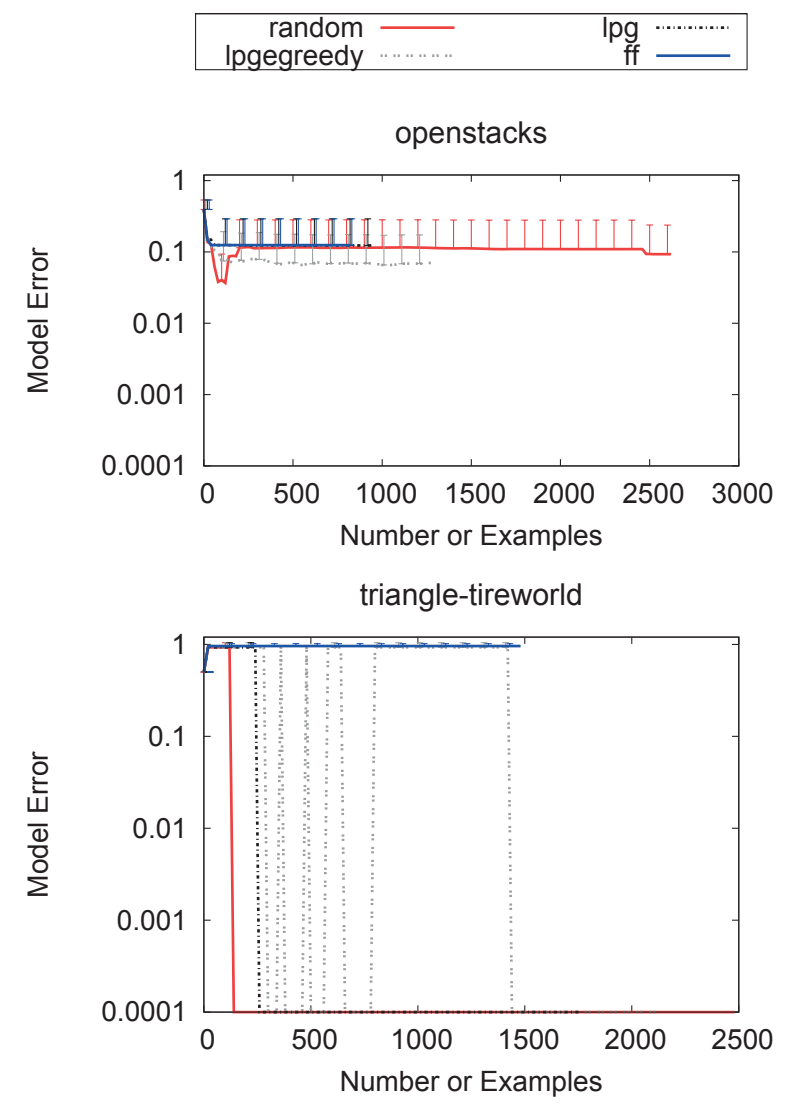

FIGURE 11. Model error in the Openstacks and the Triangle-tireworld domains. dencies.

Overall, the random strategy does not explore actions that involve strong causal depen-

These actions present preconditions that can only be satisfie by specifi sequences of actions which have low probability to be chosen by chance.

Besides, random strategies generate a greater number of learning examples because random selection of actions is not a valid strategy for solving planning problems. Hence, the random strategy (and sometimes also the $L P G-\varepsilon$ Greedy) exhausts the limit of actions for addressing the training problems. This effect is more visible in domains with dead-ends. In these domains $F F$ and $L P G$ generate fewer examples from the training problems because they usually produce execution dead-ends. On the other hand, one can use a planner for exploring domains with strong causality dependencies. However, as shown experimentally by the FF strategy, deterministic planners present a strong bias and in many domains the bias keeps execution contexts unexplored. Even more, in domains with presence of execution dead-ends in the shortest plans, this strategy may not be able to explore some actions, though they are considered in the plans.

\subsection{Pela Offlin Performance}

This experiment evaluates the planning performance of the action models learned offlin by PELA. In the offlin setup of PELA the collection of examples and the action modeling are 


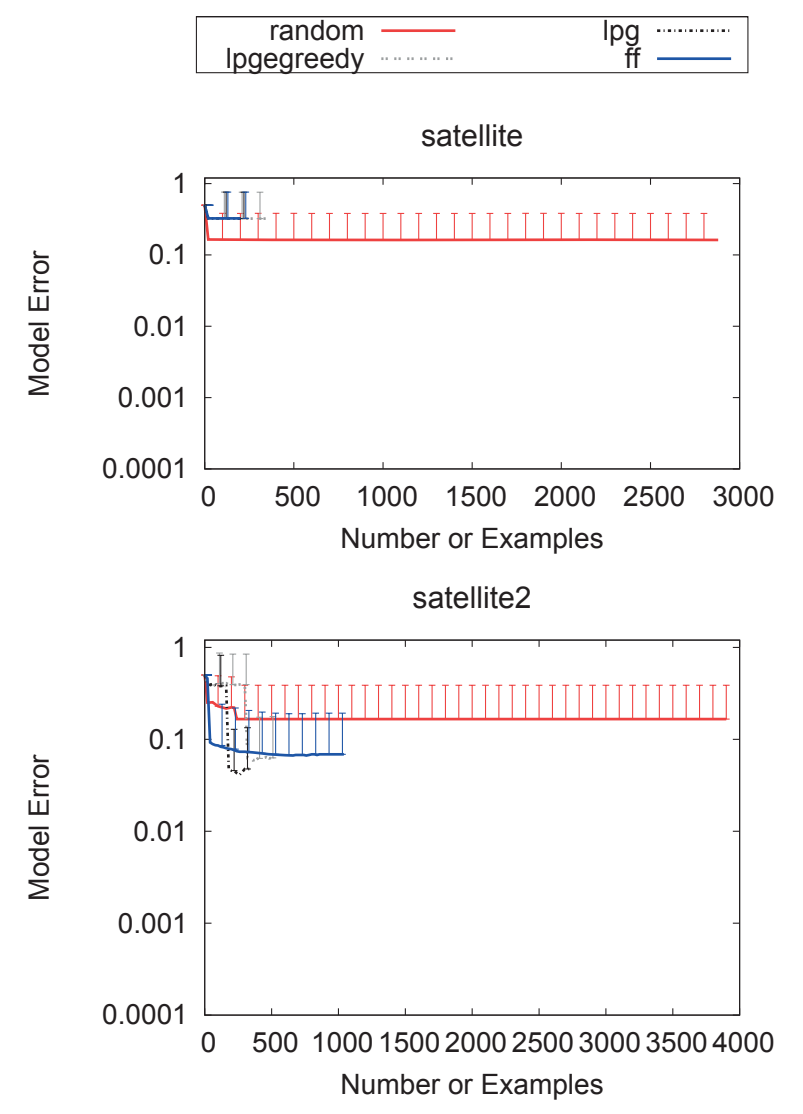

FIGURE 12. Error of the learned models in the Satellite domain.

separated from the problem solving process. This means that the updated action models are not used for collecting new observations.

The experiment is designed as follows: for each domain, PELA solves 50 small training problems and learns a set of decision trees that capture the actions performance. Then PELA compiles the learned trees into a new action model and uses the new action model to address a test set of 15 planning problems of increasing difficult . Given that the used domains are stochastic, each planning problem from the test set is addressed 30 times. The experiment compares the performance of four planning configurations

(i) FF + STRIPS model. This configuratio represents the classical replanning approach in which no learning is performed and serves as the baseline for comparison. In more detail, FF plans with the PDDL STRIPS-like action model and replans to overcome unexpected states. This configuratio (Yoon, Fern, and Givan 2007) corresponds to the best overall performer at the probabilistic tracks of IPC-2004 and IPC-2006.

(ii) FF + PELA metric model. In this configuration Metric-FF plans with the model learned and compiled by PELA. Model learning is performed after the collection of 1,000 execution episodes by the $L P G-\varepsilon$ Greedy strategy. The learned model is compiled into a metric representation (Section 3.2.1). 
(iii) GPT + PELA probabilistic model. GPT is a probabilistic planner (Bonet and Geffner 2004) for solving MDPs specifie in the high-level planning language PPDDL. GPT implements a deterministic heuristic search over the state space. In this configuratio GPT plans with the action model learned and compiled by PELA. This configuratio uses the same models than the previous configuratio but, in this case, the learned models are compiled into a probabilistic representation (Section 3.2.2).

(iv) GPT + Perfect model. This configuratio is hypothetical given that in many planning domains, the perfect probabilistic action model is unavailable. So, this config uration only serves as a reference to show how far is PELA from the solutions found with a perfect model. In this configuration the probabilistic planner GPT plans with the exact PPDDL probabilistic domain model.

Even if PELA learned perfect action models, the optimality of the solutions generated by PELA depends on the planner used for problem solving. PELA addresses problem solving with suboptimal planners because its aim is solving problems. Solutions provided by suboptimal planners cannot be proven to be optimal so we have no measure of how far PELA solutions are from the optimal ones. Nevertheless, as it is shown at IPC, suboptimal planners success to address large planning tasks achieving good quality solutions.

In the Blocksworld domain, the configuration based on the deterministic planning $(F F+$ STRIPS model and $F F+$ PELA metric model $)$ solve all the problems in the time limit (1,000 seconds). On the contrary, configuration based on probabilistic planning do not solve problems 10,14, and 15 because considering the diverse probabilistic effects of actions boosts planning complexity. In terms of planning time, planning with the actions models learned by PELA generate plans that fail less often and require less replanning episodes. In problems where replanning is expensive, that is, in large problems (problems 9 to 15), this effect means less planning time. Figure 13 shows the results obtained by the four planning configuration in the Blocksworld domain. The training set consisted of 55-blocks problems. The test set consisted of fi e 8-blocks problems, fi e 12-blocks problems, and fi e 16-blocks problems.

In the Slippery-gripper domain the $F F+$ STRIPS model configuratio is not able to solve all problems in the time limit. Because this configuratio prefers short plans, it tends to skip the dry action. As a consequence, planning with the STRIPS model fails more often and requires more replanning episodes. In problems where replanning is expensive, this configuratio exceeds the time limit. Alternatively, the configuration that plan with the models learned by PELA include the dry action because this action reduces the fragility of plans. Consequently, plans fail less often, less replanning episodes take place and less planning time is required.

In the Rovers domain, the probabilistic planning configuration are not able to solve all the problems because they handle more complex action models and consequently they scale worse. In terms of planning time, planning with the learned models is not always better (problems $7,12,13,15$ ). In this domain, replanning without the fragility metric is very cheap and it is worthy even if it generates fragile plans that fail very often. Figure 14 shows the results obtained by the four planning configuration in the Slippery-gripper and the Rovers domain. The training set for the Slippery-gripper consisted of 55-blocks problems. The test set consisted of fi e 8-blocks problems, fi e 12-blocks problems, and fi e 16-blocks problems. The training set for the Rovers domain consisted of 60 problems of 10 locations and 3 objectives. The test set consisted of 5 problems of 5 objectives and 15 locations, 5 problems of 6 objectives and 20 locations, and 5 problems of 8 objectives and 15 locations.

In the Openstacks domain, planning with the STRIPS model solves no problem. In this domain the added macro-action for making products may produce execution dead-ends. 


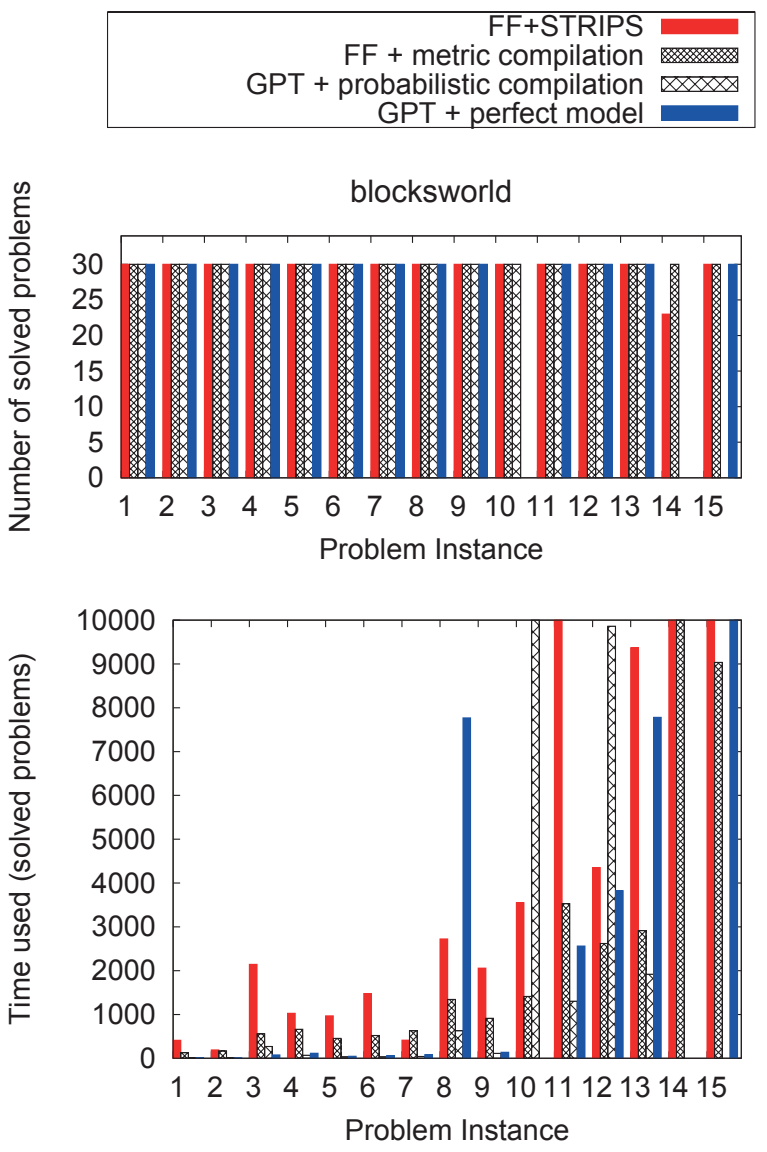

FIGURE 13. Offlin performance of PELA in the Blocksworld.

Given that the deterministic planner FF prefers short plans, it tends to systematically select this macro-action and consequently, it produces execution dead-ends. On the contrary, models learned by PELA capture this knowledge about the performance of this macro-action so it is able to solve problems. However, they are not able to reach the performance of planning with the perfect model. Though the models learned by PELA correctly capture the performance of actions, they are less compact than the perfect model so they produce longer planning times. Figure 15 shows the results obtained in the Openstacks domain.

In the Triangle-tireworld robust plans move the car only between locations with spare tires available despite these movements mean longer plans. The STRIPS action model ignores this knowledge because it assumes the success of actions. On the contrary, PELA correctly captures this knowledge learning from plans execution and consequently, PELA solves more problems than the classical replanning approach. In terms of time, planning with the models learned by PELA means longer planning times than planning with the perfect models because the learned models are less compact.

In the Satellite domain planning with the STRIPS model solves no problem. In this domain the application of action take-image without calibrating the instrument of the satellite may produce an execution dead-end. However, this model assumes that actions always succeed and as FF tends to prefer short plans, it skips the action calibrate. Therefore, 

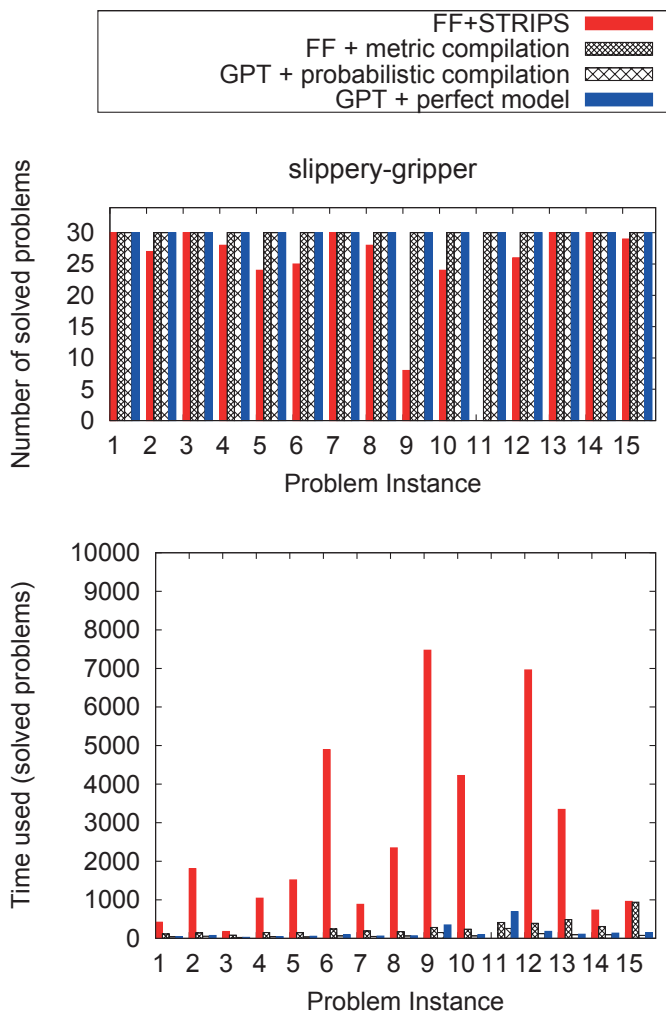
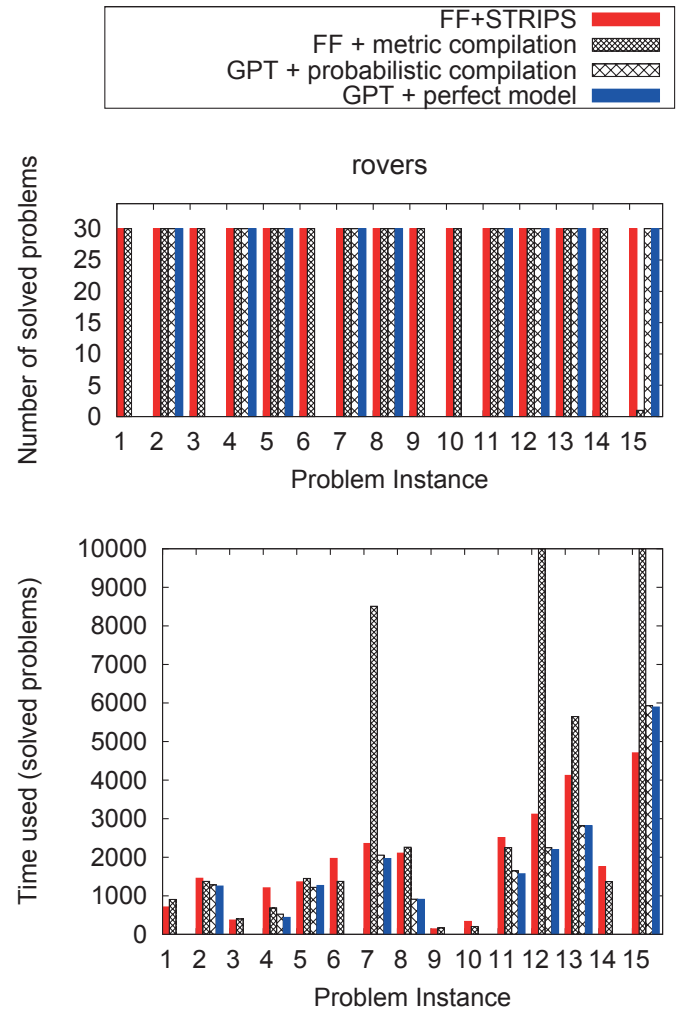

FIGURE 14. Offlin performance of PELA in the Slippery-gripper and the Rovers domains.

it generates fragile plans that can lead to execution dead-ends. Figure 16 shows the results obtained in the Triangle-tireworld and Satellite domain. The training set for the Openstacks consisted of 100 problems of 4 orders, 4 products, and 6 stacks. The test set consisted of 5 problems of 10 orders, 10 products and 15 stacks; 5 problems of 20 orders, 20 products and 25 stacks and 5 problems of 25 orders, 25 products, and 30 stacks. The training set for the Triangle-tireworld consisted of 100 problems of size 5. The test set consisted of 15 problems of increasing size ranging from size 2 to size 16.

To sum up, in dead-ends free domains planning with the models learned by PELA takes less time to solve a given problem when replanning is expensive, that is, in large problems or in hard problems (problems with strong goals interactions). In domains with presence of execution dead-ends, planning with the models learned by PELA solves more problems because dead-ends are skipped when possible. Otherwise, probabilistic planning usually yields shorter planning times than a replanning approach. Once a probabilistic planner find a good policy, then it uses the policy for all the attempts of a given problem. However, probabilistic planners scale poorly because they handle more complex action models that produce greater branching factors during search. On the other hand a classical planner needs to plan from scratch to deal with the unexpected states in each attempt. However, because they ignore diverse action effects and probabilities, they generally scale better. Table 2 summarizes the number of problems solved by the four planning configuration in the different domains. For each domain, each configuratio attempted 30 times 15 problems of increasing difficult (450 problems per domain). Table 3 summarizes the results obtained in terms of computation 

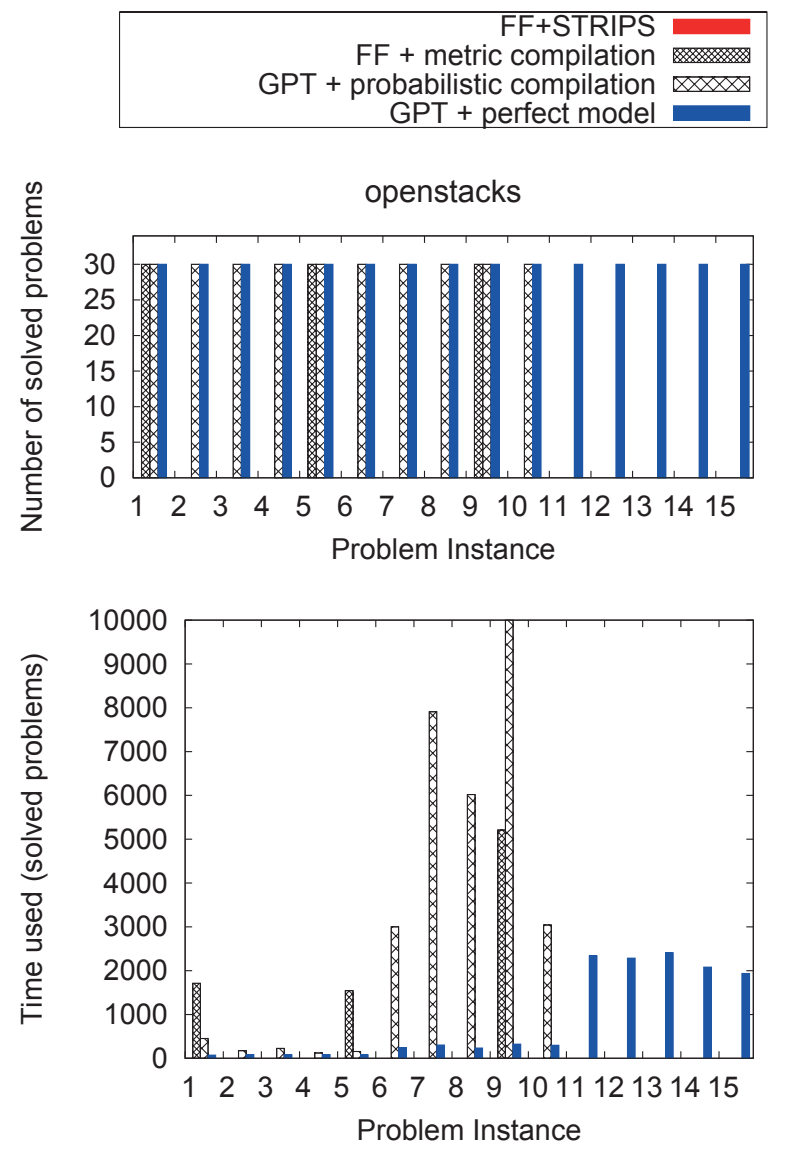

FIGURE 15. Offlin performance of PELA in the Openstacks domain.

time in the solved problems by the four planning configuration in the different domains. Both tables show results split in two groups: domains without execution dead-ends (Blocksworld, Slippery-gripper, and Rovers) and domains with execution dead-ends (OpenStacks, Triangletireworld, and Satellite).

The performance of the different planning configuration is also evaluated in terms of actions used to solve the problems. Figure 17 shows the results obtained according to this metric for all the domains. Though this metric is computed at the probabilistic track of IPC, comparing the performance of probabilistic planners regarding the number of actions is tricky. In probabilistically interesting problems, robust plans are longer than fragile plans. When this is not the case, that is, robust plans correspond to short plans, then a classical replanning approach that ignores probabilities will fin robust solutions in less time than a standard probabilistic planner because it handles simpler action models.

\subsection{Pela Online Performance}

This experiment evaluates the planning performance of the models learned by PELA within an online setup. 

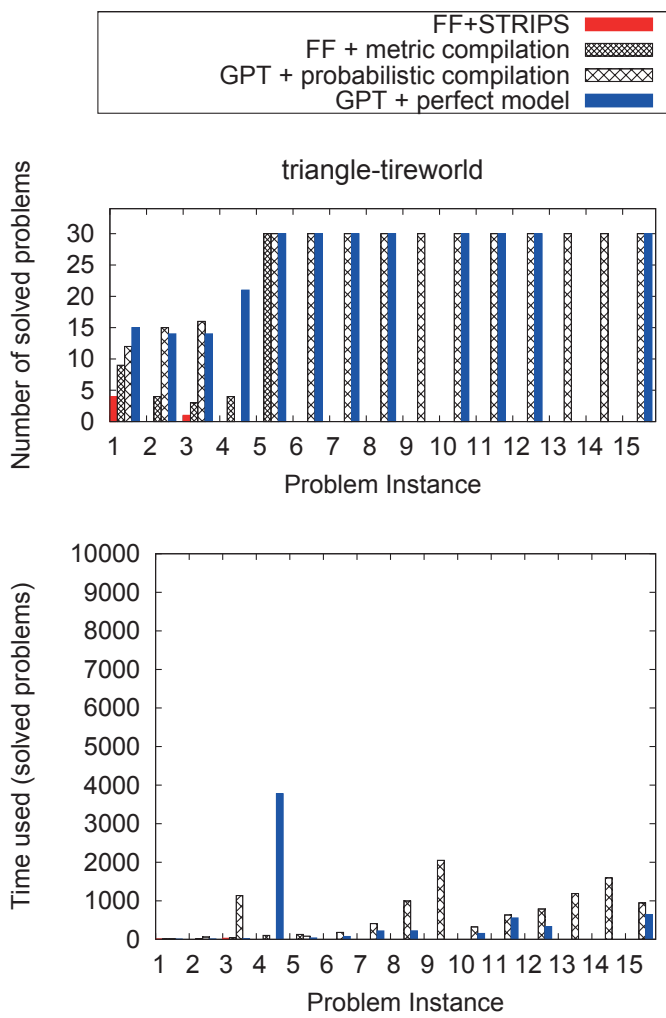
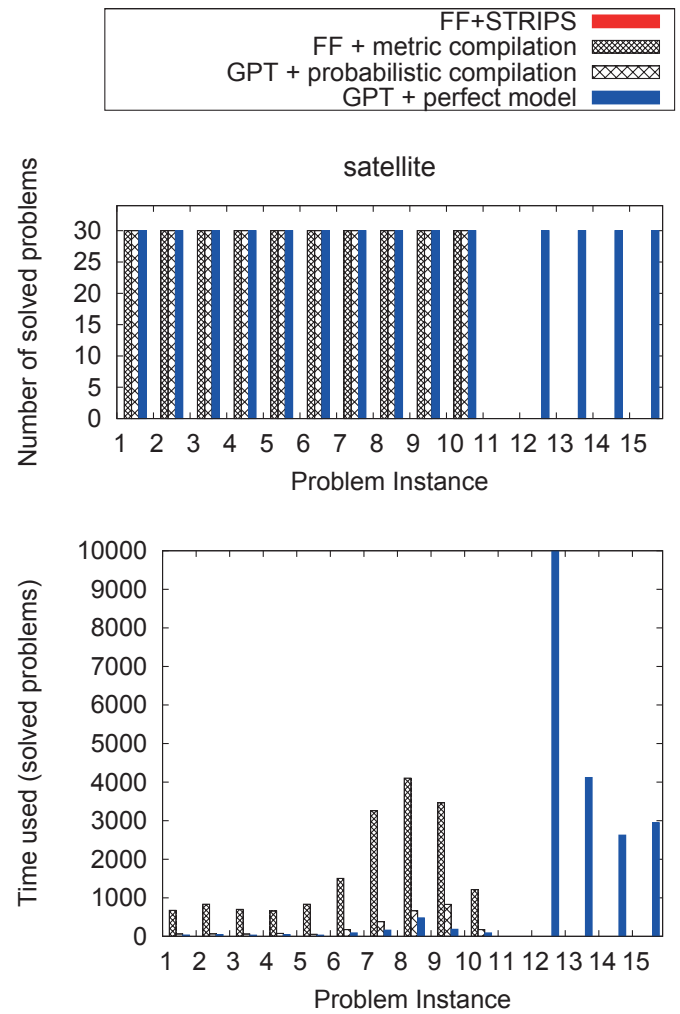

FIGURE 16. Offlin performance of PELA in the Triangle-tireworld and Satellite domains.

TABLE 2. Summary of the Number of Problems Solved by the Offlin Configuration of PELA.

\begin{tabular}{lcccc}
\hline & \multicolumn{3}{c}{ Number of problems solved } \\
\cline { 2 - 5 } & $F F$ & $\begin{array}{c}F F+\text { metric } \\
\text { model }\end{array}$ & $\begin{array}{c}G P T+\text { probabilistic } \\
\text { model }\end{array}$ & $\begin{array}{c}G P T+\text { perfect } \\
\text { model }\end{array}$ \\
\hline Blocksworld (450) & 443 & 450 & 390 & 390 \\
$\begin{array}{l}\text { Slippery-gripper } \\
\quad(450)\end{array}$ & 369 & 450 & 450 & 450 \\
Rovers (450) & 450 & 421 & 270 & 270 \\
OpenStacks (450) & 0 & 90 & 300 & 450 \\
Triangle-tireworld & 5 & 50 & 373 & 304 \\
$\quad(450)$ & & & & 420 \\
Satellite (450) & 0 & 300 & 300 & \\
\hline
\end{tabular}

The online setup of PELA consists of a closed loop that incrementally upgrades the planning action model of the architecture as more execution experience is available. In this setup PELA uses the updated action models for collecting new observations.

The experiment is designed as follows: PELA starts planning with an initial STRIPS-like action model and every 50 action executions, PELA upgrades its current action model. At 
TABLE 3. Summary of the Planning Time (Accumulated) Used by the Four Offlin Configuration of PELA.

Planning time of problems solved (seconds)

\begin{tabular}{lrrrr}
\cline { 2 - 5 } & \multicolumn{1}{c}{$F F$} & $\begin{array}{c}F F+\text { metric } \\
\text { model }\end{array}$ & $\begin{array}{c}G P T+\text { probabilistic } \\
\text { model }\end{array}$ & $\begin{array}{c}G P T+\text { perfect } \\
\text { model }\end{array}$ \\
\hline Blocksworld & $78,454.6$ & $35,267.1$ & $26,389.4$ & $38,416.7$ \\
Slippery-gripper & $36,771.1$ & 4302.7 & 1238.3 & 2167.1 \\
Rovers & $28,220.0$ & $349,670.0$ & $18,635.0$ & $18,308.9$ \\
OpenStacks & 0.0 & 8465.3 & $33,794.6$ & $12,848.7$ \\
Triangle-tireworld & 34.0 & 306.0 & $10,390.1$ & 6034.1 \\
Satellite & 0.0 & $17,244.1$ & 2541.3 & $21,525.9$ \\
\hline
\end{tabular}

each upgrade step, the experiment evaluates the resulting action model over a test set of 30 problems.

The experiment compares the performance of the two baselines described in the previous experiment $(F F+$ STRIPS and $G P T+$ Perfect model $)$ against fi e configuration of the PELA online setup. Given that the baselines implement no learning, their performance is constant in time. On the contrary, the online configuration of PELA vary their performance as more execution experience is available. These fi e online configuration of PELA are named $F F$ $\varepsilon$ Greedy and present $\varepsilon$ values of $0,0.25,0.5,0.75$, and 1.0 , respectively. Accordingly, actions are selected by the planner FF using the current action model with probability $\varepsilon$ and actions are selected randomly among the applicable ones with probability $1-\varepsilon$. These configuration range from $F F-\varepsilon$ Greed 0.0 , a fully random selection of actions among the applicable ones, to $F F-\varepsilon$ Greedy 1.0, an exploration fully guided by FF with the current action model. The $F F$ $\varepsilon$ Greedy1.0 configuratio is different from the $F F+$ STRIPS offlin configuratio because it modifie its action model with experience.

These configuration are an adaptation of the most basic exploration/exploitation strategy in RL to the use of off-the-shelf planners. RL presents more complex ways of exploring in which selection probabilities are weighted by their relative value functions. For an updated survey see Wiering (1999) and Reynolds (2002).

In the Blocksworld the fi e online configuration of PELA achieve action models able to solve the test problems faster than the classical replanning approach. In particular, except for the pure random configuratio (FF-EGreedy0.0), all PELA configuration achieve this performance after one learning iteration. This effect is because of two factors: (1) in this domain the knowledge about the success of actions is easy to capture because it is not state-dependent; and (2) in this domain it is not necessary to capture the exact probability of success of actions for robust planning; it is enough to capture the differences between the probability of success of actions that handle blocks and actions that handle towers of blocks.

In the Slippery-gripper domain, the convergence of the PELA configuration is slower. In fact, the $F F-\varepsilon G r e e d y 1.0$ PELA configuratio is not able to solve the test problems in the time limit until completing the fourth learning step. In this domain, the probability of success of actions is more difficul to capture because it is state-dependent. However, when the PELA configuration properly capture this knowledge, they need less time than the classical replanning approach to solve the test problems because they require less replanning episodes. 

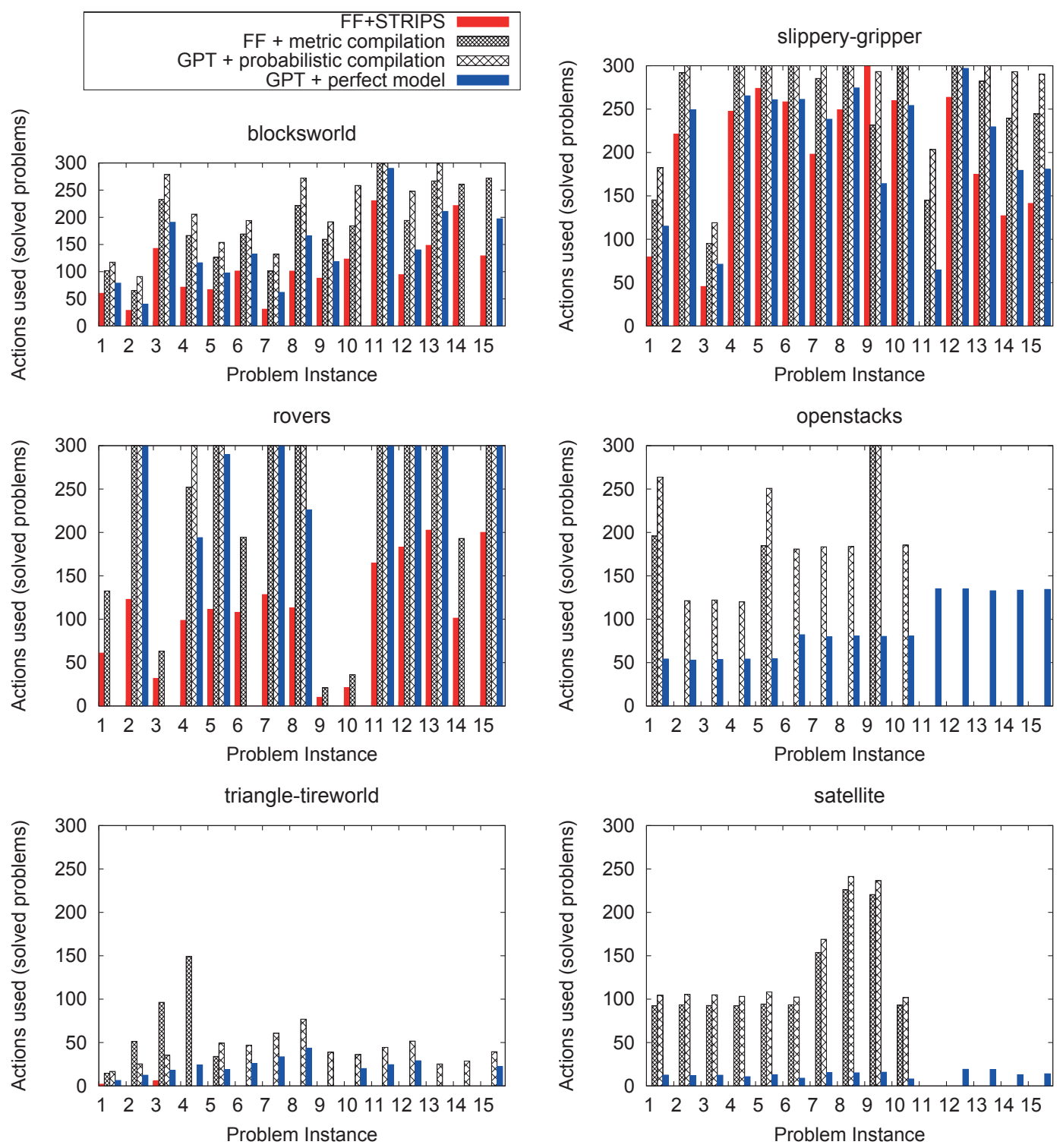

FIGURE 17. Actions used for solving the problems by the offlin configuration of PELA.

In the Rovers domain the performances of planning with STRIPS-like and planning with perfect models are very close because in this domain there is no execution dead-ends and replanning is cheap in terms of computation time. Accordingly, there is not much benefi on upgrading the initial STRIPS-like action model. Figure 18 shows the results obtained by the online configuration of PELA in the Rovers domain.

In the Openstacks domain the $F F+$ Strips baseline does not solve any problem because it generates plans that do not skip the execution dead-ends. On the contrary, all the online configuration of PELA achieve action models able to solve the test problems after one learning step. In terms of planning time, planning with the PELA models spends more time than planning with the perfect models because they are used in a replanning approach. 

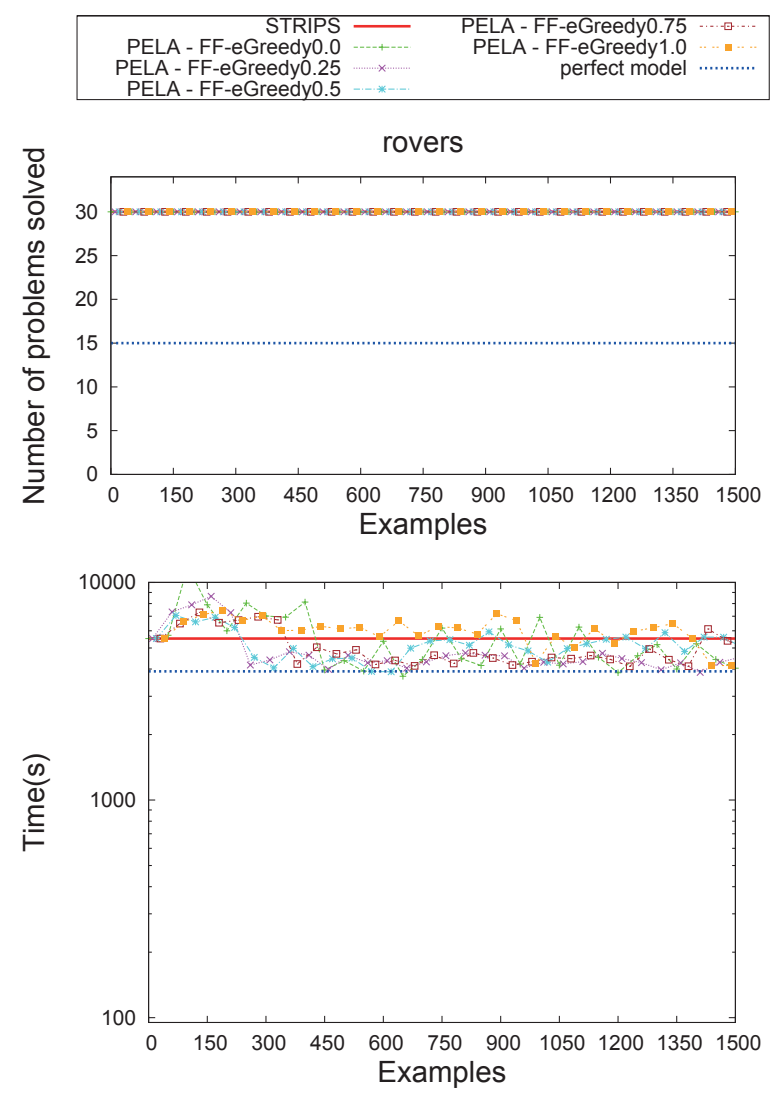

FIGURE 18. Online performance of PELA in the Rovers domain.

In the Triangle-tireworld, the $F F-\varepsilon$ Greedy 1.0 configuratio is not able to solve more problems than a classical replanning approach because it provides learning examples that always correspond to the shortest paths in the triangle. Though the PELA configuration solve more problems than a classical replanning approach, it is far from planning with the perfect model because FF does not guarantee optimal plans.

In the Satellite domain only the $F F-\varepsilon$ Greedy1.0 configuratio is able to solve the test problems because the strong causal dependencies of actions of the domain. These config urations are the only ones capable of capturing the fact that take-image may produce execution dead-ends when instruments are not calibrated. Figure 19 shows the results obtained in the Satellite domain.

Overall, the upgrade of the action model performed by PELA does not affect to actions causality. Therefore, the online configuratio of PELA can assimilate execution knowledge without degrading the coverage performance of a classical replanning approach. In particular, experiments show that even at the firs steps of the online learning process (when the learned knowledge is imperfect), the introduction of the learned knowledge does not prevent PELA to solve problems. On the other hand, approaches that learn probabilistic action models from scratch by generalizing human provided observations (Pasula et al. 2007) cannot guarantee the soundness of their intermediate action models. In particular, these approaches cannot guarantee that a given set of learning examples will produce an action model able to solve problems with a fi ed planning strategy. 

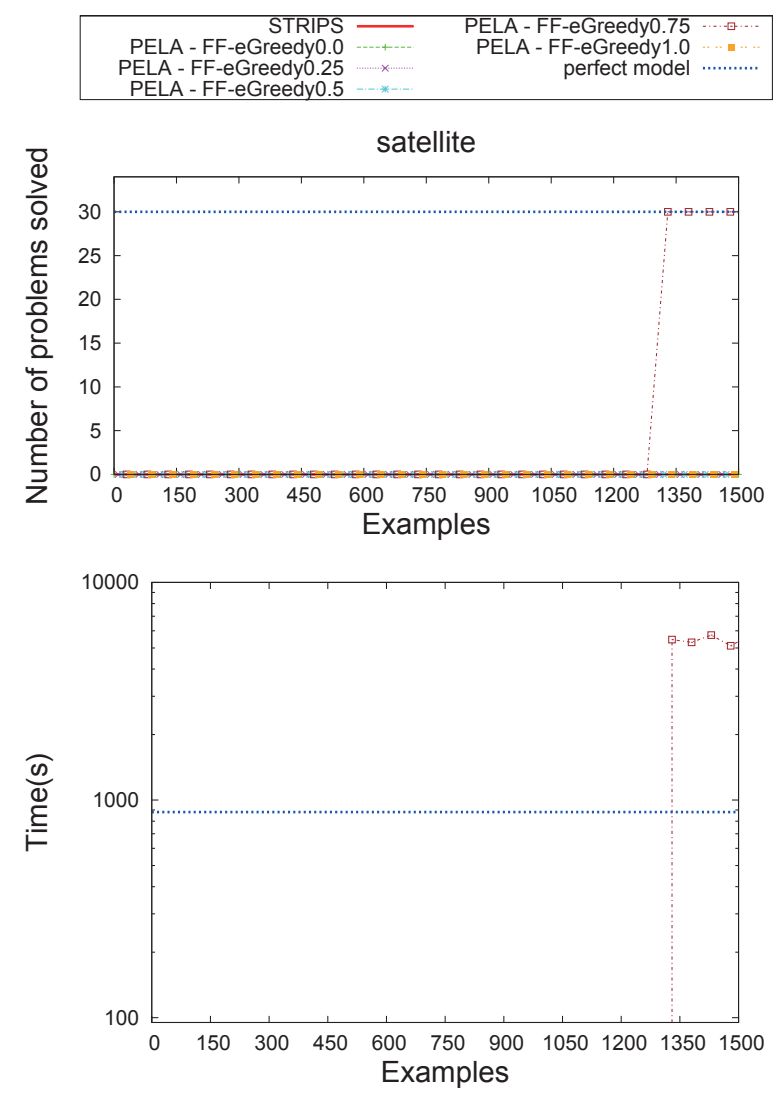

FIGURE 19. Online performance of PELA in the Satellite domain.

Besides, once PELA is presented with enough execution experience, the PELA online configuration address probabilistic planning problems more robustly than the classical replanning approach. Nevertheless, the action models learned within the online setup may not properly capture the performance of all actions in a given domain. Execution experience may be insufficien (generally at the firs learning steps) or too biased (the training problems may provide learning examples of the same kind). As shown experimentally, these problems are more noticeable in domains with execution dead-ends. In these domains, the performance of the PELA online configuration depend on capturing some key actions, that is, the actions that produce execution dead-ends. When a given configuratio does not capture the success of the key actions it will perform poorly. On the other hand, this effect is less noticeable in domains free from execution dead-ends. In this kind of domains, configuration can outperform a classical replanning approach though the success of actions is not exactly captured. Table 4 summarizes the number of problems solved by the seven planning configuration in the different domains at the end of the online learning process. For each domain, each configuratio attempted 30 problems of increasing difficult . Table 5 summarizes the results obtained in terms of computation time in the solved problems by the seven planning configuration in the different domains. Both tables show results split in two groups: domains without execution dead-ends (Blocksworld, Slippery-gripper, and Rovers) and domains with execution dead-ends (OpenStacks, Triangle-tireworld, and Satellite). The number of problems solved is not revealing in domains without dead-ends, because the seven configuration 
TABLE 4. Summary of the Number of Problems Solved by the Online Configuration of PELA.

\begin{tabular}{lccccccc}
\hline & \multicolumn{5}{c}{ Number of problems solved at the end of the online process } \\
\cline { 2 - 7 } & Strips & $\varepsilon$ Greedy0.0 & $\varepsilon$ Greedy0.25 & $\varepsilon$ Greedy0.5 & $\varepsilon$ Greedy0.75 & EGreedy1.0 & Perfect model \\
\hline Blocksworld (30) & 30 & 30 & 30 & 30 & 30 & 30 & 30 \\
Slippery-gripper (30) & 30 & 30 & 30 & 30 & 30 & 30 & 30 \\
Rovers (30) & 30 & 30 & 30 & 30 & 30 & 30 & 15 \\
OpenStacks (30) & 0 & 30 & 30 & 30 & 30 & 30 & 30 \\
Triangle-tireworld (30 & 0 & 2 & 0 & 1 & 30 & 0 & 15 \\
Satellite (30) & 0 & 0 & 0 & 0 & 0 & 30 \\
\hline
\end{tabular}

TABLE 5. Summary of the Computation Time (Accumulated) Used by the Four Online Configuration of PELA.

\begin{tabular}{lccccccc}
\hline & \multicolumn{7}{c}{ Planning time in the solved problems at the end of the online process } \\
\cline { 2 - 7 } & Strips & $\varepsilon$ Greedy0.0 & $\varepsilon$ Greedy0.25 & $\varepsilon$ Greedy0.5 & $\varepsilon$ Greedy0.75 & $\varepsilon$ Greedy1.0 & Perfect model \\
\hline Blocksworld & 2094.4 & 1183.8 & 1372.6 & 989.4 & 1137.6 & 1056.0 & 308.0 \\
Slippery-gripper & 497.6 & 968.2 & 424.6 & 436.6 & 423.0 & 415.0 & 102.2 \\
Rovers & 5522.2 & 4037.4 & 4526.0 & 5003.4 & 4992.0 & 4233.8 & 3906.2 \\
OpenStacks & 0 & $13,527.4$ & $12,221.4$ & $12,808.4$ & $13,399.6$ & $12,936.0$ & 1323.4 \\
Triangle-tireworld & 0 & 258.0 & 0 & 50.0 & 0 & 0 & 1976.0 \\
Satellite & 0 & 0 & 0 & 0 & $5,730.4$ & 0 & 881.0 \\
\hline
\end{tabular}

solve all the problems. In this kind of domains one must analyze the planning time, given that fragile plans imply more replanning episodes are needed, and consequently longer planning times. On the contrary, the number of problems solved is a reliable performance measure in domains with execution dead-ends.

\section{RELATED WORK}

There is extensive prior work on general architectures for reasoning, execution and learning, ranging from execution oriented, as in robotics applications (Peterson and Cook 2003), to more cognitive oriented (Rosenbloom, Newell, and Laird 1993). The most relevant example to our work is ROGUE (Haigh and Veloso 1999) which learned propositional decision trees and used them as control rules for the PRODIGY planning architecture (Veloso et al. 1995). These architectures are not based on standard languages like PDDL or PPDDL for reasoning and learning, and different planning or learning techniques cannot be easily plugged-in and tested over a variety of domains.

The firs approach for the PELA (Jimenez, Fernandez, and Borrajo 2005) captured the performance of instantiated actions as control rules for the Prodigy planner (Veloso et al. 1995). A second approach (Jimenez, Fernandez, and Borrajo 2008), closer to the current architecture also learned relational trees about the performance of actions. This approach did not implement $\varepsilon$ Greedy strategies for the online integration of planning and learning and lacked of exhaustive evaluation.

There are recent works that also study how to automatically enrich planning action models. In many applications it is difficul to code PDDL actions for modeling motions: the geometry of the environment may be complex to analyze, the duration of these actions may be difficul to be define, etc. Instead, these works automatically verify and build planning actions of this kind using knowledge from motion plans (Choi and Amir 2009; Wolfe, Marthi, and Russell 2010). 


\subsection{Action Modeling}

Focusing on the action modeling, we classifie the existing learning approaches according to two features (1) the kind of learned models (deterministic vs. probabilistic) and (2) the observability of the state of the environment (partial vs. full).

Despite other classification are possible, for instance the target of the learning (preconditions, effects, conditions of effects, probabilities of outcomes, etc.) we believe this one is useful for planning purposes because each class corresponds to a different planning paradigm.

5.1.1. Learning Deterministic Actions in Fully Observable Environments. This problem is closely related to the Inductive Logic Programming (ILP) problem. In this regard, the hot spots for planning are how to generate significan learning examples (how to explore the performance of planning actions) and how to handle defective learned knowledge (how to plan when the learned action models are incomplete and incorrect). The LIVE system (Shen and Simon 1989) alternated problem solving with rule learning for the automatic definitio of STRIPS-like operators. The decision for alternation mainly depends on surprises, that is, situations where an action's consequences violate its predicted models. When no rule can be found for solving the problem, LIVE will generate and execute an exploration plan, or a sequence of actions, seeking for surprises to extend the rule set. The EXPO system (Gil 1992) refine incomplete planning operators, that is, operators with some missing preconditions and effects. EXPO generates plans, monitors their execution, and detects differences between the state predicted according to the internal action model and the observed state. EXPO constructs a set of specifi hypotheses to fi the detected differences. After being heuristically filtere, each hypothesis is tested in turn with an experiment and a plan is constructed to achieve the situation required to carry out the experiment.

OBSERVER (Wang 1994), unlike previous works that refine planning operators by an active exploration of the environment, learned operators by observing expert agents. The observations of the expert agent consists of: (1) the sequence of actions being executed, (2) the prestate and the poststate resulting from the execution of each action. OBSERVER learned planning operators from these observation sequences in an incremental fashion using a conservative specific-to-genera inductive generalization process. Eventually, the system solves practice problems with the new operators to refin them from execution traces. The LOPE system (Garcia-Martinez and Borrajo 2000) learned planning operators by observing the consequences of executing planned actions in the environment. At the beginning, the system has no knowledge, it perceives the initial situation, and selects a random action to execute in the environment. Then it loops by (1) executing an action, (2) perceiving the resulting situation of the action execution and its utility, (3) learning a model from the perception and (4) planning for further interaction with the environment (in case the execution of the plan is finishe, or the system has observed a mismatch between the predicted situation and the situation perceived). The planning component of LOPE does not explicitly receive a goal input given that LOPE creates its own goals from the situations with the highest utility.

Because in this category the effects of actions are deterministic and fully observable, they can be acquired by lifting the delta-state (the set of literals that differ between the prestate and the posstate) of an action execution. In this regard, the main difficult is to extract the actual preconditions of actions, because the direct lifting of a prestate may include unnecessary preconditions. Recently, the work reported in Walsh and Littman (2008) succeeds to bound the number of interactions the learner must have with the environment to learn the preconditions (and effects) of a STRIPS action model. 
5.1.2. Learning Deterministic Actions in Partially Observable Environments. This category, given that observations of the current state are incomplete, requires ILP techniques able to deal with noise in the learning examples. In this category, one can fin two different approaches. On the one hand, the ARMS system (Yang, Wu, and Jiang 2007) which encodes example plan traces as a weighted maximum satisfiabilit problem, from which a candidate STRIPS-like action model is extracted. The output of ARMS is a single model, which is built heuristically in a hill-climbing fashion. Consequently, the resulting model is sometimes inconsistent with the input.

On the other hand, Amir and Chang introduced an algorithm that exactly learns all the STRIPS-like models that could have lead to a historical of observations (Amir and Chang 2008). Given a formula representing the initial belief state, a sequence of executed actions $\left(a_{1}, a_{2}, \ldots, a_{n}\right)$ and the corresponding observed states $\left(s_{1}, \ldots, s_{n}\right)$, the learning algorithm updates the formula of the belief state with every action and observation in the sequence. This update makes sure that the new formula represents exactly all the transition relations that are consistent with the actions and observations. The formula returned at the end includes all consistent models, which can be retrieved then with additional processing. Both techniques do not consider the exploration process needed to extract the learning examples and assume they are provided by an external expert.

\subsubsection{Learning Probabilistic Actions in Fully Observable Environments. The PELA} architecture fit in this category.

The task addressed in this category does not need to handle sets of possible states because they are fully observable. On the contrary, actions present stochastic effects, so they can not be learned by just lifting the delta-state. This task is very much related with the induction of stochastic logic models like Stochastic Logic Programs (Cussens 2001; Muggleton 2001), Bayesian Logic Programs (Jaeger 1997; Kersting and Raedt 2001) or Markov Logic Networks (Richardson and Domingos 2006).

One of the earliest works of this kind was the TRAIL system (Benson 1997) that used Inductive Logic Programming to learn operators for reactive behavior. Besides preconditions and postconditions, these operators contained a success rate that indicated the percentage of times when the operator successfully achieved its postcondition.

The most relevant work in this category (Pasula et al. 2007) is able to learn from scratch more expressive action models than PELA including preconditions and different outcomes of actions. However, this approach does not generate its own learning examples and requires specifi planning and learning algorithms. Instead, PELA explores the world to generate its own learning examples, captures uncertainty of the environment using existing standard machine learning techniques and compiles it into standard planning models that can be directly fed into different kinds of off-the-shelf planners (like cost-based or probabilistic planners). S, PELA can directly profi from the last advances in both field without modifying the source of the architecture. Even more, the off-the-shelf spirit of the architecture allows PELA to change the learning component to acquire other useful planning information, like actions duration (Lanchas et al. 2007).

5.1.4. Learning Probabilistic Actions in Partially Observable Environments. Further studies are needed for action modeling in stochastic and partially observable environments. Preliminary work (Yoon and Kambhampati 2007) addresses this problem using techniques for weighted maximum satisfiabilit in order to fin the action model that better explains the collected observations. 


\subsection{RL}

RL agents interact with the environment to collect experience which, by means of appropriate algorithms, is processed to generate an optimal policy (Kaelbling et al. 1996). RL includes two different approaches:

- Model-Based RL uses a model of the environment to generate advise on how to explore it, so that the agent can fin better policies. When model-based techniques use a relational representation (Croonenborghs et al. 2007), they produce action models similar to the ones learned by PELA. In this case, the main difference comes from the fact that PELA handles action models that follow the standard planning representation languages PDDL/PPDDL and that PELA delegates problem solving to off-the-shelf classical/probabilistic planners.

- Model-Free RL does not benefi from a model of the environment. In some domains there is so much uncertainty that learning to achieve goals is easier than learning accurate action models. Accordingly, model-free RL algorithms do not model the decision-making as a function of the state, like value/heuristic functions, but as a function of pairs $<$ state, action $>$ called action-value functions. The q-function is an example of an action-value function which provides a measure of the expected reward for taking action $a$ in state $s$ performance measure. Relational representations of the q-function (Dzeroski, Raedt, and Driessens 2001) adopt the same representation as symbolic planning to efficient y code the function in relational space states.

The aims of RL are closely related to the aims of PELA. In fact model-Based Relational RL (RRL) techniques succeed to address some planning problems. Normally, these techniques rely on complete state enumeration and their time complexity is polynomial in the size of the state-space. In planning problems, the size of the state-space grows exponentially with the number of features describing the problem (objects properties and objects relations). Overcoming the state-space explosion of model-Based RL in planning problems is an interesting research line. A promising research direction is using heuristic search algorithms to limit computation to the states reachable from the initial state. Besides, these algorithms can benefi from domain-independent heuristics extracted from the planning problem representation, like the FF heuristic (Hoffmann and Nebel 2001). This research line includes the LAO* algorithm (Hansen and Zilberstein 2001), a generalization of the $\mathbf{A}^{*}$ algorithm for MDPs, or the Learning Depth-First Search (LDFS) algorithm (Bonet and Geffner 2006), a generalization of the IDA* for MDPs.

Model-Free RRL was also able to learn good policies for some type of planning problems like building a tower of blocks. However, more research is needed to efficient y address problems with highly interacting goals. For instance, when building a tower of specifi blocks on $(V, W)$, on $(W, X)$, on $(X, Y)$, on $(Y, Z)$. This is the kind of problems traditionally addressed in automated planning where the achievement of a particular goal may undo previously satisfie goals. In these problems, model-Free RRL often spends a long time exploring regions of the state-action space without learning anything because no rewards (goal states) are encountered. To provide model-Free RRL with some positive rewards and relieve the limitations of random exploration, recent works exploit human-define policies (Driessens and Matwin 2004) or transfer learning (Croonenborghs, Driessens, and Bruynooghe 2007).

Besides, learning techniques for planning (Zimmerman and Kambhampati 2003) try to learn general knowledge useful when solving any problem of a given domain. Learning of 
generalized policies (Khardon 1999; Martin and Geffner 2000; Winner and Veloso 2003) is an example of capturing this general knowledge. Model-Free RL focuses learning on addressing particular problems so each time the type of the problem changes model-Free $\mathrm{RL}$ agents need learning from scratch, or at least a transfer learning process (Fernández and Veloso 2006; Torrey et al. 2007; Mehta et al. 2008; Taylor and Stone 2009). When using a relational representation model-Free RRL can solve problems of the same type with additional objects without reformulating their learned policies, although additional training may be necessary to achieve optimal (or even acceptable) performance levels.

Works on RL study the convergence of the evaluation function as more experience is available. This function combines action model and problem solving information. PELA follows greedy strategies for both, learning and problem solving, trading off optimality in exchange for scalability. In terms of model learning, PELA uses techniques for learning relational decision trees. Because of the huge size of the search space handled by these techniques PELA uses greedy search and heuristic pruning techniques that succeed to induce compact rule sets in a reasonable time. These rule sets are not proved to be optimal, because the exhaustive search of this search space is intractable. In terms of problem solving, there are two approaches in automated planning for guaranteeing optimality:

- Exploring the whole search space. Unfortunately, state spaces in planning are normally huge producing combinatorial explosion.

- Following an $\mathbf{A}^{*}$ algorithm that explores the search space guided by an admissible heuristic. Unfortunately, admissible heuristics for planning are poorly informed, so optimal planners are only able to solve small problem instances. In any case, since we compile into two different planning schemes, advances in any of those can automatically improve planning under uncertainty as understood in this paper.

\section{CONCLUSION AND FUTURE WORK}

This paper describes the PELA architecture for robust planning in nondeterministic domains. In order to achieve robust plans within these domains, PELA automatically upgrades an initial STRIPS like planning model with execution knowledge of two kinds: (1) probabilistic knowledge about the success of actions and (2) predictions of execution dead-ends. Moreover, the upgrade of the action models performed by PELA does not affect the actions causality and hence, it is suitable for online integration of planning and learning. The PELA architecture is based on off-the-shelf planning and learning components and uses standard representation languages like PDDL or PPDDL. Therefore, different planning and/or learning techniques can be directly plugged-in without modifying the architecture.

The performance of the architecture has been experimentally evaluated over a diversity of probabilistic planning domains:

- The model correctness experiments revealed that random explorations of planning domains improve the accuracy of the learned models because they explore the performance of actions in diverse contexts. However, pure random explorations are inappropriate for planning domains with strong causal dependencies. Random explorations do not explore actions that require the execution of a fi ed sequence of steps to be applicable. In these domains, the use of planners with stochastic behavior (like LPG or $\varepsilon$ Greedy strategies) provide diversity to the learning examples, as well as considering causal dependencies of actions. 
- The offlin performance experiments showed that the action models learned by PELA make both, metric-based and probabilistic planners, generate more robust plans than a classical replanning approach. In domains with execution dead-ends, planning with the models learned by PELA increases the number of solved problems. In domains without execution dead-ends, planning with the models learned by PELA is beneficia when replanning is expensive. On the other hand, the action models learned by PELA increase the size of the initial STRIPS model, meaning generally longer planning times. Specifical y, the increase in size is proportional to the number of leaf nodes of the learned trees. One can control the size of the resulting trees by using declarative biases as the amount of tree pruning desired. However, extensive pruning may result in a less robust behavior as leaf nodes would not be so fin grained.

- The online performance experiments showed that the upgrade of the action models proposed by PELA does not affect to the actions causality and consequently, it is suitable for an online integration of planning and learning. Even at the firs learning steps, in which the gathered experience is frequently scarce and biased, the performance of PELA is not worse than a classical replanning approach. When the gathered experience achieves enough quality, PELA addresses probabilistic planning problems more robustly than the classical replanning approach.

Currently we are working on using PELA to acquire more execution information useful for planning. An example is our previous work on learning actions durations (Lanchas et al. 2007). Other interesting direction is learning dead-deads information with knowledge about the goals and including it in the planning models. With this regard a possible direction is using the plan constraints and preferences define at PDDL3.0 (Gerevini et al. 2009).

Our current version of PELA assumes there is an initial action model of the environment which correctly and completely captures the nominal effects of actions. However, in complex or changing environments, even definin simple STRIPS-like action models may become a hard task. We have an evident interest in relaxing this assumption. Recently, Kambhampati introduced the concept of model-lite planning (Kambhampati 2007) for encouraging the development of planning techniques that do not search for a solution plan but for the most plausible solution plan that respects the current action model. New work on approximate inference (Yoon and Kambhampati 2007; Thon, Landwehr, and Raedt 2008; Lang and Toussaint 2009) seems to be a way of approaching the development of these new planning techniques. In addition the evaluation of PELA assumed full observability of the environment states. However, observations of the real world may provide state representations of the environment that are incomplete or incorrect. Given that decision trees deal with noisy learning examples, a natural extension of this work is the study of the PELA performance in environments where observations provide states with noisy information.

Contrary to intuition, we have found that it is not always worthy to generate a very accurate and complete action model, because its expressiveness sometimes does not pay off the complexity of planning with it. As an example, FF-replan was the best performer using a STRIPS model of actions for solving problems with probabilistic encodings at IPC-2004 and IPC-2006. In this way, further research is needed to determine in which situations learning a complex model and planning with it is better than learning a simple model and planning with it.

\section{REFERENCES}

AMIR, EYAL, and A. CHANG. 2008. Learning partially observable deterministic action models. Journal of Artificia Intelligence Research, 33:349-402. 
Benson, ScotT SherwOod. 1997. Learning Action Models for Reactive Autonomous Agents. Ph.D. dissertation, Stanford University, Palo Alto, CA.

BlockeEl, HendriK, and LuC De RAedt. 1998. Top-down induction of first-orde logical decision trees. Artificia Intelligence, 101(1-2):285-297.

BONET, BLAI, and HÉCTOR GEFFNER. 2004. mgpt: A probabilistic planner based on heuristic search. Journal of Artificia Intelligence Research, 24:933-944.

BONET, BLAI, and HeCtOR GEFFNER. 2006. Learning depth-firs search: A unifie approach to heuristic search in deterministic and non-deterministic settings, and its application to MDPs. In International Conference on Automated Planning and Scheduling, ICAPS06.

CHOI, JAESIK, and EyAL AMIR. 2009. Combining planning and motion planning. In ICRA'09: Proceedings of the 2009 IEEE international conference on Robotics and Automation, IEEE Press, Piscataway, NJ. pp. $4374-4380$.

Croonenborghs, Tom, Kurt Driessens, and Maurice Bruynooghe. 2007. Learning relational options for inductive transfer in relational reinforcement learning. In Proceedings of the Seventeenth Conference on Inductive Logic Programming.

Croonenborghs, Tom, Jan Ramon, Hendrik Blockeel, and Maurice Bruynooghe. 2007. Online learning and exploiting relational models in reinforcement learning. In Proceedings of the 20th International Joint Conference on Artificia Intelligence, AAAI Press, pp. 726-731.

CUSSENS, JAMES. 2001. Parameter estimation in stochastic logic programs. Machine Learning, 44(3):245-271.

DRIESSENS, KURT, and STAN MATWIN. 2004. Integrating guidance into relational reinforcement learning. Machine Learning, 57:271-304.

Dzeroski, SAso, Luc De Raedt, and Kurt Driessens. 2001. Relational reinforcement learning. Machine Learning, 43:7-52.

FernÁndez, Fernando, and MANUela Veloso. 2006. Probabilistic policy reuse in a reinforcement learning. In International conference on Autonomous Agents and Multiagent Systems (AAMAS).

Fox, MAria, Alfonso Gerevini, Derek Long, and Ivan Serina. 2006. Plan stability: Replanning versus plan repair. In International Conference on Automated Planning and Scheduling (ICAPS'06).

FoX, M., and D. LONG. 2003. PDDL2.1: An extension to PDDL for expressing temporal planning domains. Journal of Artificia Intelligence Research, 20:61-124.

Garcia-MARTinez, RAMON, and DANiEl BorRajo. 2000. An integrated approach of learning, planning, and execution. Journal of Intelligent and Robotics Systems, 29:47-78.

Gerevini, Alfonso, Alessandro Saetti, and IVAn Serina. 2003. Planning through stochastic local search and temporal action graphs in LPG. Journal of Artificia Intelligence Research, 20:239-290.

Gerevini, Alfonso E., Patrik Haslum, Derek Long, Alessandro Saetti, and Yannis Dimopoulos. 2009. Deterministic planning in the fift international planning competition: PDDL3 and experimental evaluation of the planners. Artificia Intelligence, 173(5-6):619-668.

Ghallab, Malik, Dana Nau, and Paolo Traverso. 2004. Automated Planning Theory and Practice. Morgan Kaufmann: San Mateo, CA.

GIL, YolandA. 1992. Acquiring Domain Knowledge for Planning by Experimentation. Ph.D. dissertation, School of Computer Science, Carnegie Mellon University, Pittsburgh, PA.

Haigh, Karen Zita, and Manuela M. Veloso. 1999. Learning situation-dependent rules. In AAAI Spring Symposium on Search Techniques for Problem Solving under Uncertainty and Incomplete Information.

HANSEN, ERIC A., and Shlomo Zilberstein. 2001. LAO: A heuristic search algorithm that find solutions with loops. Artificia Intelligence, 129(1-2):35-62.

HOFFMANN, J. 2003. The metric-FF planning system: Translating ignoring delete lists to numerical state variables. Journal of Artificia Intelligence Research, 20:291-341.

HofFMAnn, JörG, and BERnHARD NeBEl. 2001. The FF planning system: Fast plan generation through heuristic search. JAIR, 14:253-302. 
JAEGER, MANFRED. 1997. Relational bayesian networks. In Conference on Uncertainty in Artificia Intelligence.

Jiménez, Sergio, Fernando FernÁndez, and Daniel Borrajo. 2005. Machine learning of plan robustness knowledge about instances. In European Conference on Machine Learning.

Jiménez, Sergio, Fernando Fernández, and DAniel Borrajo. 2008. The pela architecture: integrating planning and learning to improve execution. In National Conference on Artificia Intelligence (AAAI'2008).

Kaelbling, Leslie Pack, Michael L. Littman, and Andrew P. Moore. 1996. Reinforcement learning: A survey. Journal of Artificia Intelligence Research, 4:237-285.

KAmbHampati, SubBaraO. 2007. Model-lite planning for the web age masses: The challenges of planning with incomplete and evolving domain models. In Senior Member track of the AAAI, Seattle, WA.

Kersting, KRISTIAN, and LUC DE RAEDT. 2001. Towards combining inductive logic programming with Bayesian networks. In International Conference on Inductive Logic Programming, pp. 118-131.

KHARDON, RONI. 1999. Learning action strategies for planning domains. Artificia Intelligence, 113:125-148.

Lanchas, Jesús, Sergio Jiménez, Fernando Fernández, and Daniel Borrajo. 2007. Learning action durations from executions. In Workshop on AI Planning and Learning. ICAPS'07.

LANG, TOBIAS, and MARC TOUSSAINT. 2009. Approximate inference for planning in stochastic relational worlds. In International Conference on Machine Learning.

LitTle, IAIN, and Sylvie Thí́BAUX. 2007. Probabilistic planning vs replanning. In Workshop on International Planning Competition: Past, Present and Future. ICAPS07.

MARTIN, M., and H. GEFFNER. 2000. Learning generalized policies in planning using concept languages. In International Conference on Artificia Intelligence Planning Systems, AIPS00.

Mehta, Neville, Sriraam Natarajan, Prasad Tadepalli, and Alan Fern. 2008. Transfer in variable-reward hierarchical reinforcement learning. Machine Learning, 73(3):289-312.

Muggleton, S.H. 2001. Stochastic logic programs. In New Generation Computing. Academic Press: New York.

Pasula, Hanna M., Luke S. Zettlemoyer, and Leslie Pack Kaelbling. 2007. Learning symbolic models of stochastic domains. Journal of Artificia Intelligence Research, 29:309-352.

PETERSON, G.L., and D.J. CoOK. 2003. Incorporating decision-theoretic planning in a robot architecture. Robotics and Autonomous Systems, 42(2):89-106.

QUINLAN, J.R. 1986. Induction of decision trees. Machine Learning, 1(1):81-106.

Reynolds, Stuart IAn. 2002. Reinforcement Learning with Exploration. Ph.D. thesis, The University of Birmingham, Birmingham, UK.

Richardson, MATthew, and Pedro Domingos. 2006. Markov logic networks. Machine Learning, 62:107-136.

Rosenbloom, P. S., A. Newell, and J. E. Laird. 1993. Towards the knowledge level in Soar: the role of the architecture in the use of knowledge. MIT Press: Cambridge, MA.

SHEN, W.M., and H.A. SIMON. 1989. Rule creation and rule learning through environmental exploration. In International Joint Conference on Artificia Intelligence, IJCAI-89.

TAYLOR, MATtheW E., and PETER StONE. 2009. Transfer learning for reinforcement learning domains: A survey. Journal of Machine Learning Research, 10(1):1633-1685.

Thon, Ingo, Niels Landwehr, and Luc De Raedt. 2008. A Simple Model for Sequences of Relational State Descriptions. In European Conference on Machine Learning.

Torrey, LisA, Jude W. SHAVLIK, TREVOR WALKER, and RiCHARD MACLIN. 2007. Relational macros for transfer in reinforcement learning. In Conference on Inductive Logic Programming, pp. 254-268.

Veloso, M., J. Carbonell, A. Pérez, D. Borrajo, E. FinK, and J. Blythe. 1995. Integrating planning and learning: The PRODIGY architecture. JETAI, 7(1):81-120.

Walsh, Thomas J., and Michael L. LitTMAn. 2008. Efficien learning of action schemas and web-service descriptions. In AAAI'08: Proceedings of the 23rd national conference on Artificia intelligence, AAAI Press. pp. $714-719$. 
WANG, XUEMEI. 1994. Learning planning operators by observation and practice. In International Conference on AI Planning Systems, AIPS-94.

WIERING, MARCO. 1999. Explorations in efficien reinforcement learning. Ph.D. thesis, University of Amsterdam IDSIA, the Netherlands.

WINNER, ELLY, and MANUELA Veloso. 2003. DISTILL: Towards learning domain-specifi planners by example. In International Conference on Machine Learning, ICML'03.

WOLFE, JASON, BHASKARA MARTHI, and STUART RUSSELL. 2010. Combined task and motion planning for mobile manipulation. In International Conference on Automated Planning and Scheduling, Toronto, Canada.

YANG, QIANG, KANGHENG WU, and YUNFEI JiANG. 2007. Learning action models from plan traces using weighted max-sat. Artificia Intelligence Journal, 171:107-143.

YoOn, SungWOOK, ALAN Fern, and Bob GIVAN. 2007. Ff-replan: A baseline for probabilistic planning. In International Conference on Automated Planning and Scheduling (ICAPS '07).

YoOn, SUNGWOOK, and SUBBARAO KAMBHAMPATI. 2007. Towards model-lite planning: A proposal for learning and planning with incomplete domain models. In ICAPS2007 Workshop on Artificia Intelligence Planning and Learning.

Younes, H.L.S., M. L. LitTMan, D. Weissman, and J. Asmuth. 2005. The firs probabilistic track of the international planning competition. Journal of Artificia Intelligence Research, 24:851-887.

ZIMMERMAN, TERRY, and SUBBARAO KAMBHAMPATI. 2003. Learning-assisted automated planning: looking back, taking stock, going forward. AI Magazine, 24:73-96. 